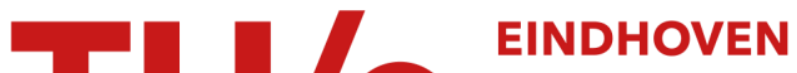 UNIVERSITY OF TECHNOLOGY
}

\section{Langevin and diffusion equation of turbulent fluid flow}

Citation for published version (APA):

Brouwers, J. J. H. (2010). Langevin and diffusion equation of turbulent fluid flow. Physics of Fluids, 22(8), 085102-1-16. [085102]. https://doi.org/10.1063/1.3466658

DOI:

10.1063/1.3466658

Document status and date:

Published: 01/01/2010

\section{Document Version:}

Publisher's PDF, also known as Version of Record (includes final page, issue and volume numbers)

\section{Please check the document version of this publication:}

- A submitted manuscript is the version of the article upon submission and before peer-review. There can be important differences between the submitted version and the official published version of record. People interested in the research are advised to contact the author for the final version of the publication, or visit the $\mathrm{DOI}$ to the publisher's website.

- The final author version and the galley proof are versions of the publication after peer review.

- The final published version features the final layout of the paper including the volume, issue and page numbers.

Link to publication

\section{General rights}

Copyright and moral rights for the publications made accessible in the public portal are retained by the authors and/or other copyright owners and it is a condition of accessing publications that users recognise and abide by the legal requirements associated with these rights.

- Users may download and print one copy of any publication from the public portal for the purpose of private study or research.

- You may not further distribute the material or use it for any profit-making activity or commercial gain

- You may freely distribute the URL identifying the publication in the public portal.

If the publication is distributed under the terms of Article 25fa of the Dutch Copyright Act, indicated by the "Taverne" license above, please follow below link for the End User Agreement:

www.tue.nl/taverne

Take down policy

If you believe that this document breaches copyright please contact us at:

openaccess@tue.nl

providing details and we will investigate your claim. 


\title{
Langevin and diffusion equation of turbulent fluid flow
}

\author{
J. J. H. Brouwers \\ Eindhoven University of Technology, P.O. Box 513, 5600 MB Eindhoven, The Netherlands
}

(Received 27 January 2010; accepted 26 May 2010; published online 11 August 2010)

\begin{abstract}
A derivation of the Langevin and diffusion equations describing the statistics of fluid particle displacement and passive admixture in turbulent flow is presented. Use is made of perturbation expansions. The small parameter is the inverse of the Kolmogorov constant $C_{0}$, which arises from Lagrangian similarity theory. The value of $C_{0}$ in high Reynolds number turbulence is 5-6. To achieve sufficient accuracy, formulations are not limited to terms of leading order in $C_{0}^{-1}$ including terms next to leading order in $C_{0}^{-1}$ as well. Results of turbulence theory and statistical mechanics are invoked to arrive at the descriptions of the Langevin and diffusion equations, which are unique up to truncated terms of $\mathcal{O}\left(C_{0}^{-2}\right)$ in displacement statistics. Errors due to truncation are indicated to amount to a few percent. The coefficients of the presented Langevin and diffusion equations are specified by fixed-point averages of the Eulerian velocity field. The equations apply to general turbulent flow in which fixed-point Eulerian velocity statistics are non-Gaussian to a degree of $\mathcal{O}\left(C_{0}^{-1}\right)$. The equations provide the means to calculate and analyze turbulent dispersion of passive or almost passive admixture such as fumes, smoke, and aerosols in areas ranging from atmospheric fluid motion to flows in engineering devices. (c) 2010 American Institute of Physics.
\end{abstract}

[doi:10.1063/1.3466658]

\section{INTRODUCTION}

An old problem of turbulent fluid flow is the statistical description of turbulent dispersion. A rudimentary approach is to model turbulent dispersion analogous to molecular diffusion. It results in the widely used semiempirical equation of turbulent diffusion (see Ref. 1, Chap. 5, where various references to other works in this field can be found). The coefficients of diffusion in the semiemperical equation of turbulent diffusion are of an approximate nature. They are based on Reynolds analogy, phenomenological considerations, and adjustment to measurements. Moreover, there are doubts whether the formulation of the equation itself is valid. ${ }^{1-3}$ Stochastic theory provides a derivation of the diffusion equation under homogeneous or almost homogeneous conditions only. However, the important cases of turbulent flow are known to be strongly inhomogeneous. Fixed-point (Eulerian) statistical averages of fluid velocities and energy dissipation rate vary considerably in space. It is unclear whether the concept of a diffusion equation holds under such circumstances $^{1-3}$ let alone the specification of the turbulent diffusion coefficients.

A more fundamental approach is one which starts from a Langevin equation to describe the statistical behavior of the velocity of a fluid particle. The description is analogous to the fluctuation equations formulated for macroscopic variables in molecular dynamics. ${ }^{4-7}$ The approach dates back to the work of Taylor. ${ }^{8}$ Important advantage of this method is that it is consistent with the asymptotic structure of turbulent flow at large Reynolds number. The Langevin equation for fluid particle can be made to match the inertial subrange limit of the Lagrangian version of Kolmogorov's similarity theory. ${ }^{2,9,10}$ The problem, however, is the form of the damping function to be used in the Langevin equation. While for homogeneous isotropic turbulence, the damping function is well-established, ${ }^{9-11}$ a unique expression valid for general anisotropic inhomogeneous turbulence is missing. Several expressions have been proposed, e.g., Refs. 9, 10, 12, and 13, but the validity or range of applicability is limited or left unspecified.

The starting point of the present analysis is to describe turbulent dispersion through a Langevin equation. The inverse of the Kolmogorov constant $C_{0}$, which emerges from Lagrangian similarity theory, is introduced as a small parameter. It is used to develop a solution procedure, which is based on perturbation expansions involving powers of $C_{0}^{-1}$. Invoking results of turbulence theory and statistical physics, a unique formulation of the Langevin equation and its damping function is derived. The diffusion equation follows from the long-time limit of the Langevin equation. The coefficients of the Langevin and diffusion equations are described in terms of fixed-point (Eulerian) statistical averages of the flow field. The descriptions hold for general forms of turbulence in which fixed-point Eulerian velocity statistics are non-Gaussian to a degree of $\mathcal{O}\left(C_{0}^{-1}\right)$. The descriptions entail a truncation error of $\mathcal{O}\left(C_{0}^{-2}\right)$ in dispersion statistics. It is indicated that this is expected to lead to errors of only a few percent.

\section{LANGEVIN EQUATION}

A major achievement in turbulence theory is Kolmogorov's description of the statistical structure of the small viscous scales of turbulence. ${ }^{14,15}$ The description holds for large Reynolds numbers $\operatorname{Re}=u_{*} L \nu^{-1}$, where $u_{*}$ is the typical value of fluctuating fluid velocity, $\nu$ is the kinematic viscosity, and $L$ is the external length scale, e.g., radius of a pipe in which turbulent flow takes place, height of a channel, 
thickness of a turbulent boundary layer, etc. Several elements of Kolmogorov's universal theory have in the meantime been confirmed by the experiment, ${ }^{14,16}$ as well as by theoretical analysis $^{15}$ and direct numerical simulation (DNS) of the Navier-Stokes equations. ${ }^{10}$ Measurements and results of DNS of more recent date lend support to the Lagrangian interpretation of Kolmogorov's theory. ${ }^{16}$ One of the outcomes of the Lagrangian theory of small scales is the tendency of fluid particle accelerations toward a delta-correlated process. Correlation times of fluid particle accelerations decrease as $\mathrm{Re}^{-1 / 2}$ when compared to those of fluid particle velocities, a relationship confirmed by experiment. ${ }^{16}$ It gives rise to the idea to describe the statistical process of fluid particle velocity by a Langevin equation

$$
\frac{d v_{i}^{\prime}}{d t}=a_{i}^{\prime}\left(\boldsymbol{v}^{\prime}, \boldsymbol{x}\right)+\left\{C_{0} \varepsilon(\boldsymbol{x})\right\}^{1 / 2} w_{i}(t), \quad i=1,2,3,
$$

where $v_{i}^{\prime}=v_{i}^{\prime}(t)$ is the statistical representation of fluctuating fluid particle velocity relative to Eulerian mean velocity $u_{i}^{0}(\boldsymbol{x})$ evaluated at particle position $\boldsymbol{x}=\boldsymbol{x}(t)$. Velocity is related to position by

$$
\frac{d x_{i}}{d t}=u_{i}^{0}(\boldsymbol{x})+v_{i}^{\prime}
$$

In Eq. (1), $a_{i}^{\prime}=a_{i}^{\prime}\left(\boldsymbol{v}^{\prime}, \boldsymbol{x}\right)$ is the damping function, $C_{0}$ is the universal Kolmogorov constant, $\varepsilon=\varepsilon(\boldsymbol{x})$ is the energy dissipation rate averaged at fixed position, and $w_{i}(t)$ is the Gaussian white noise of unit intensity. The turbulent flow field is taken to be stationary in the fixed inertial $(x, t)$ frame, implying that statistical averages of flow variables at fixed positions $\boldsymbol{x}$ do not vary with time. Equations (1) and (2) can be solved for various realizations of $w_{i}(t)$, taking appropriate conditions at $t=0$. The statistics of velocity and position of a tagged fluid particle or passive fluid admixture can then be assessed by averaging the resulting time records at fixed moments in time. An alternative method to obtain the same statistical information is to solve the Fokker-Planck equation for the joint probability of particle velocity and position associated with Eqs. (1) and (2).

The observation that second order correlations of fluid particle accelerations tend to those of a delta-correlated process when $\operatorname{Re} \gg 1$ is in itself not sufficient to justify the Langevin model. The description of the forcing term by Gaussian white noise resorts to applying ordinary nonintermittent Kolmogorov (K-41) theory. ${ }^{17}$ Effects of intermittency, ${ }^{18}$ apparent in corrections in higher order structure functions, are not accounted for in the Langevin model. For that purpose, one can adopt a fractal model based on Kolmogorov's refined similarity theory. ${ }^{19,20}$ However, statistical averages of particle displacement, which determine turbulent dispersion, are seen to change little under such approach. ${ }^{19,20}$ The effect of intermittency is apparent in the small viscous scales, which govern the acceleration process, rather than in the large energetic scales, which govern the velocity process of turbulence. ${ }^{15}$ It is for these reasons that a Langevin model resting on K-41 theory is considered to be a sound approach for modeling dispersion.
To predict the statistics of particle velocities and positions using Eqs. (1) and (2), descriptions are needed for $u_{i}^{0}(\boldsymbol{x}), \varepsilon(\boldsymbol{x})$, and $a_{i}^{\prime}\left(\boldsymbol{v}^{\prime}, \boldsymbol{x}\right)$. There are several routes to values of fixed-point (Eulerian) averages of velocity $u_{i}^{0}(\boldsymbol{x})$ and of energy dissipation rate $\varepsilon(\boldsymbol{x})$. Depending on the flow configuration under consideration, one can apply techniques of DNS of the Navier-Stokes equations, results of measurements, methods of computational fluid dynamics, and results of theoretical analysis. What is missing is a sound and general description of the damping function $a_{i}^{\prime}(\boldsymbol{v}, \boldsymbol{x})$. It is only for homogeneous isotropic Gaussian turbulence that the damping function has been formally established. ${ }^{9-11}$ For practically relevant nonhomogeneous turbulence, several descriptions have been proposed but they rely in part on ad hoc assumptions and their status is unclear. The objective of the subsequent analysis is to derive a unique as possible description of the damping function. A second objective is to derive expressions from the Langevin equation, which hold for long times after particle marking leading to the diffusion equation.

\section{WELL-MIXED CONDITION}

A well-established method for constraining the form of the damping function is to implement the well-mixed condition. The condition was introduced in generalized form by Thomson who showed it to supersede several other conditions previously imposed on stochastic models of turbulent particle trajectories. ${ }^{2}$ The well-mixed condition follows from the Eulerian interpretation of the Fokker-Planck equation associated with Eqs. (1) and (2),

$$
\frac{\partial p_{E}}{\partial t^{\prime}}=\frac{1}{2} C_{0} \varepsilon \frac{\partial^{2} p_{E}}{\partial u_{i}^{\prime} \partial u_{i}^{\prime}}-\frac{\partial}{\partial u_{i}^{\prime}}\left(a_{i}^{\prime} p_{E}\right)-u_{i}^{\prime} \frac{\partial p_{E}}{\partial x_{i}^{\prime}},
$$

where $p_{E}=p_{E}\left(u^{\prime}\right)$ is the probability density of the fluctuating component $\boldsymbol{u}^{\prime}$ of Eulerian fluid velocity determined at a fixed point in a fixed inertial frame. For convenience, in subsequent mathematical treatments, we have introduced a coordinate system $\left(x^{\prime}, t^{\prime}\right)$, which moves with the mean velocity $\boldsymbol{u}^{0}$,

$$
\frac{d x_{i}}{d t^{\prime}}=u_{i}^{0}, \quad x_{i}=x_{i}^{\prime} \quad \text { at } t=0,
$$

where $x_{i}^{\prime}$ is the position in the moving system. Statistical averages involving $p_{E}\left(\boldsymbol{u}^{\prime}\right)$ are treated as functions of $\boldsymbol{x}^{\prime}$ and $t^{\prime}$. As $\partial / \partial t^{\prime}=\partial / \partial t+u_{i}^{0}\left(\partial / \partial x_{i}\right)$, such averages may be constant in the nonmoving inertial $(\boldsymbol{x}, t)$ frame but they become timedependent when described in the coordinates of the moving $\left(\boldsymbol{x}^{\prime}, t^{\prime}\right)$ system when they vary in the direction of the mean flow in the fixed system. The motivation for employing a moving coordinate system both here and in subsequent parts of the analysis is as follows. In turbulence, mean velocities tend to be much larger than fluctuating velocities. The presence of terms involving the mean velocity in the FokkerPlanck equation therefore complicates analysis of the magnitude of the various terms. A more transparent situation arises through the presented coordinate transformation. It eliminates the terms involving the mean velocity and brings the formulation of the Fokker-Planck equation closer to the variables that matter in turbulence. Rather than mean veloc- 
ity, large-scale turbulence is governed by the spatial gradients of mean velocity. Their magnitude is determined by fluctuating velocities and external lengths, which can be considered as the primary variables that characterize turbulence. The formulation of the Fokker-Planck equation and Langevin equation in the moving frame, here and in Secs. IV-VIII, gives direct insight into the effects of these primary variables on the various terms. It leads to a more transparent situation which will be exploited in setting up effective perturbation expansions.

Over the past 50 years, a lot of data have been collected on fixed-point Eulerian velocities in turbulent flow. These data indicate that in many cases, Eulerian velocity statistics are more or less Gaussianly distributed. These include turbulence behind a grid, ${ }^{21}$ turbulent pipe flow, ${ }^{22,23}$ turbulent boundary layers, ${ }^{24}$ and turbulence between counter-rotating disks. ${ }^{16}$ In all these cases, Gaussianity was observed to a degree that absolute values of skewness and kurtosis were limited to about 0.3 . Note that Gaussianity of velocity statistics is not in contradiction with the strong non-Gaussian and intermittent behavior of accelerations and spatial derivatives of velocities. ${ }^{15}$ The latter is governed by the small viscous scales of turbulence, while the contribution of the small viscous scales in the spectrum of velocities is very small at large Reynolds number. Gaussianity of fixed-point Eulerian velocities is a reasonable proposition in the light of experimental evidence. Later in this paper, we shall consider the case of light non-Gaussianity (Sec. VIII).

The zero-mean Gaussian distribution $p_{G}=p_{G}\left(\boldsymbol{u}^{\prime}\right)$ of fluctuating velocity $\boldsymbol{u}^{\prime}$ is given by

$$
p_{G}=(2 \pi)^{3 / 2} \lambda^{1 / 2} \exp \left(-\frac{1}{2} \lambda_{i j} u_{i}^{\prime} u_{j}^{\prime}\right),
$$

where $\lambda$ is the determinant of $\lambda_{i j}$, the latter being the inverse of the covariance tensor or Reynolds stress tensor,

$$
\lambda_{i j}=\sigma_{i j}^{-1},
$$

where

$$
\sigma_{i j}=\left\langle\left\langle u_{i}^{\prime} u_{j}^{\prime}\right\rangle\right\rangle=\left\langle u_{i}^{\prime} u_{j}^{\prime}\right\rangle,
$$

with $\sigma_{i j}=\sigma_{j i}$. Angled brackets are used to denote ensemble averages determined at a fixed position in the fixed inertial frame; double angled brackets denote cumulant. Fluctuating Eulerian velocities are related to total Eulerian velocity $u_{i}$ by

$$
u_{i}=u_{i}^{o}+u_{i}^{\prime}
$$

where $u_{i}^{o}=\left\langle u_{i}\right\rangle$ is the mean Eulerian velocity. In the case of stationary turbulence in the fixed inertial frame and invoking ergodicity, the above ensemble averages can be determined by time averaging of fluctuating velocities measured or calculated by DNS at fixed positions.

Substituting the Gaussian density into Eq. (3), we obtain an equation for the damping function of the form

$$
\begin{aligned}
\frac{\partial a_{i}^{\prime}}{\partial u_{i}^{\prime}}-\lambda_{i j} u_{j}^{\prime} a_{i}^{\prime}= & \frac{1}{2} C_{0} \varepsilon\left(-\lambda_{i i}+\lambda_{i k} \lambda_{i j} u_{k}^{\prime} u_{j}^{\prime}\right) \\
& -\frac{1}{2}\left(\lambda^{-1} \frac{\partial \lambda}{\partial t^{\prime}}-\frac{\partial \lambda_{i j}}{\partial t^{\prime}} u_{i}^{\prime} u_{j}^{\prime}\right) \\
& -\frac{1}{2}\left(\lambda^{-1} \frac{\partial \lambda}{\partial x_{i}^{\prime}} u_{i}^{\prime}-\frac{\partial \lambda_{k j}}{\partial x_{i}^{\prime}} u_{k}^{\prime} u_{j}^{\prime} u_{i}^{\prime}\right) .
\end{aligned}
$$

The above equation gives rise to particular solutions $a_{i}^{\prime P}$ superimposed on homogeneous solutions $a_{i}^{\prime H}$,

$$
a_{i}^{\prime}=a_{i}^{\prime H}+a_{i}^{\prime P} .
$$

The particular solutions were given by Thomson ${ }^{2}$

$$
\begin{aligned}
a_{i}^{\prime P}= & -\frac{1}{2} C_{0} \varepsilon \lambda_{i j} u_{j}^{\prime}+\frac{1}{2} \lambda_{j m} \frac{\partial \sigma_{m i}}{\partial t^{\prime}} u_{j}^{\prime}+\frac{\partial \sigma_{i k}}{\partial x_{k}^{\prime}} \\
& +\frac{1}{2} \lambda_{j n} \frac{\partial \sigma_{i j}}{\partial x_{m}^{\prime}}\left(u_{m}^{\prime} u_{n}^{\prime}-\sigma_{m n}\right),
\end{aligned}
$$

where in connection with Eqs. (9) and (11), it is noted that in view of Gaussianity $\lambda^{-1}\left(\partial / \partial t^{\prime}\right) \lambda=-\lambda_{i m}\left(\partial / \partial t^{\prime}\right) \sigma_{m i}$. The homogeneous solutions are obtained by setting the right hand side of Eq. (9) to zero

$$
\frac{\partial a_{i}^{\prime H}}{\partial u_{i}^{\prime}}-\lambda_{i j} u_{j}^{\prime} a_{i}^{\prime H}=0,
$$

or alternatively,

$$
\frac{\partial}{\partial u_{i}^{\prime}}\left(a_{i}^{\prime H} p_{G}\right)=0 .
$$

Solutions of the homogeneous problem can be linear in $u_{i}^{\prime}$ or higher order in $u_{i}^{\prime}$. The solution, which is linear in $u_{i}^{\prime}$, is given by

$$
a_{i}^{\prime H I}=b_{k} \sigma_{i l} \varepsilon_{k l j} u_{j}^{\prime},
$$

where $\varepsilon_{k l j}$ is the alternating unit tensor. In Eq. (14), $b_{k}$ are three constants whose values are unknown. Combining the above result with the linear part of the particular solution according to Eq. (11) leads to a description of the linear part of the damping function, which contains three unknown constants. It is a reflection of what is known as the nonuniqueness problem: a full statistical specification of fixed-point Eulerian velocities is insufficient to completely specify the damping function of the Langevin equation of turbulence. ${ }^{9}$

The nonuniqueness problem repeats itself in homogeneous solutions of higher order in $u_{i}^{\prime}$. The solution, which is zero-mean quadratic in $u_{i}^{\prime}$, is given by

$$
a_{i}^{\prime H I I}=\gamma_{i m n}\left(u_{m}^{\prime} u_{n}^{\prime}-\sigma_{m n}\right),
$$

where $\gamma_{i m n}=\gamma_{i n m}$. The coefficients $\gamma_{i m n}$ are determined by

$$
\begin{gathered}
\gamma_{i m n}\left(\sigma_{j m} \sigma_{l n}+\sigma_{j n} \sigma_{l m}\right)+\gamma_{j m n}\left(\sigma_{i m} \sigma_{l n}+\sigma_{i n} \sigma_{l m}\right) \\
\quad+\gamma_{l m n}\left(\sigma_{i m} \sigma_{l n}+\sigma_{i n} \sigma_{l m}\right)=0
\end{gathered}
$$

which constitute 10 independent equations for 18 unknown $\gamma_{i m n}$. It leads to expressions for $\gamma_{i m n}$ (not presented here) which contain eight unknown constants. The above result can be combined with the zero-mean quadratic part of the 
particular solution according to Eq. (11) to arrive at a zeromean quadratic representation of the damping function, which contains eight unknown constants. It illustrates once more the nonuniqueness problem.

The nonuniqueness problem vanishes if we implement the condition that the statistics of particle velocities in the three directions are isotropic in which case $a_{i}^{H}=0$ (see Ref. 11). This assumption applies to homogeneous isotropic turbulence at some distance behind a grid. However, for the more practically relevant cases of anisotropic inhomogeneous turbulent flow, the assumption that particle velocities in the three directions are independent of each other no longer holds.

A way out to nonuniqueness under anisotropic conditions could be one where Eulerian-based statistical information is not only restricted to same-time averages but where also account is taken of correlations in time between Eulerian velocities. However, this approach ends up in complexity because of the phenomenon of convection of small viscous scales by large scales. A direct and simple connection between Eulerian and Lagrangian correlations in time is only very short-lived after particle marking and cannot be applied in the inertial subrange of times. ${ }^{25}$ Other methods have to be introduced to solve the nonuniqueness problem.

\section{SCALING POSSIBILITIES}

The Langevin equation of turbulent flow follows from Eqs. (1), (10), and (11) as

$$
\begin{aligned}
\frac{d v_{i}^{\prime}}{d t^{\prime}}= & -\frac{1}{2} C_{0} \varepsilon \lambda_{i j} v_{j}^{\prime}+\frac{1}{2} \lambda_{j m} \frac{\partial \sigma_{m i}}{\partial t^{\prime}} v_{j}^{\prime}+\frac{\partial \sigma_{i k}}{\partial x_{k}^{\prime}} \\
& +\frac{1}{2} \lambda_{j n} \frac{\partial \sigma_{i j}}{\partial x_{m}^{\prime}}\left(v_{m}^{\prime} v_{n}^{\prime}-\sigma_{m n}\right)+a_{i}^{\prime H}+\left(C_{0} \varepsilon\right)^{1 / 2} w_{i}\left(t^{\prime}\right) .
\end{aligned}
$$

As we describe velocity dynamics in a frame moving with the mean flow [cf. Eq. (4)], particle position is described by the fluctuation equation

$$
\frac{d x_{i}^{\prime}}{d t^{\prime}}=v_{i}^{\prime} .
$$

Eulerian-based averages occurring as parameters in Eq. (17), i.e., $\lambda_{i j}, \sigma_{i j}$, and $\varepsilon$ are treated as functions of $\boldsymbol{x}^{\prime}$ and $t^{\prime}$.

Let us compare the magnitude of the second, third, and fourth terms with that of the first term on the right hand side of Eq. (17). A typical magnitude for the second term can be inferred from the von Kármán-Howarth equation ${ }^{1}$ for decaying turbulence behind a grid: $\left(\partial / \partial t^{\prime}\right) \sigma_{m j}=-\frac{2}{3} \varepsilon \delta_{m j}$, where $\delta_{m j}$ is Kronecker delta. It shows that the second term compares to the first as $C_{0}^{-1}$, unless the turbulent flow is stationary in the moving frame in which case the second term is zero altogether. The third and fourth terms describe the effect of covariance inhomogeneity. They will be small in locally homogeneous areas, such as in the log-layer of wall-induced turbulence where in contrast to $\varepsilon, \sigma_{i j}$ varies hardly with distance from the wall. ${ }^{26}$ However, away from the wall in the main section of wall-induced turbulence, the third and fourth terms will attain values which can be represented by $u_{*}^{2} L^{-1}$, where $u_{*}$ is the characteristic value of fluctuating velocity and $L$ is the external length scale. Noting that $\varepsilon$ scales as $u_{*}^{3} L^{-1}$, these terms compare with the magnitude of the first term as $C_{0}^{-1}$. A similar scaling result is obtained for other forms of inhomogeneous turbulence involved with shearing: Couette flow, turbulent jets and wakes, and flow between counter-rotating disks. It illustrates that there is no dimensionless combination of parameters, i.e., a dimensionless number, which can be used to compare the magnitudes of the various terms in the Langevin equation (17) for general forms of turbulence. If we make Eq. (17) dimensionless using $L$ and $L u_{*}^{-1}$ as basic quantities to scale distance and time and note that $a_{i}^{\prime H}$ scales as $u_{*}^{2} L^{-1}$, we arrive at an equation with no dimensionless number in it. The only parameter left in the dimensionless Langevin equation, which can be used to order the magnitudes of the various terms, is the Kolmogorov constant $C_{0}$. Its largeness provides a possibility to construct perturbation expansions based on powers of $C_{0}^{-1}$ which exhibit convergence and which enable simplification of the problem. The value of the Kolmogorov constant is the same for all sorts of turbulence. It is related to the small viscous scales of turbulence that tend to a universal isotropic state, which is independent of the large energetic scales of turbulence when $\operatorname{Re} \gg 1$ (see Ref. 14). In the analysis of the Langevin equation (17), the Kolmogorov constant can be treated as an independent parameter, which does not influence or is influenced by the other statistical parameters whose magnitudes are controlled by the large energetic scales of turbulence. Results of DNS and measurements obtained over the past decade reveal values of $C_{0}$ of about 6 for turbulent flow at sufficient large values of the Reynolds number. ${ }^{10}$ The corresponding value of $C_{0}^{-1}$ is 0.167 . Clearly, this value is not very small. Furthermore, because it is a constant and not a dimensionless number which can be made arbitrarily small by changing the values of its constituting parameters, truncation of higher order terms can be rather tricky. Yet the expansion schemes based on powers of $C_{0}^{-1}$ introduced in the subsequent part of this paper are found to work well. The reason is that we do not only limit our analysis to terms of leading order but also include the next to leading order terms. Truncation is applied to terms of magnitude $C_{0}^{-2}$ directly compared to the magnitude of the leading terms. Note here that when $C_{0}=6, C_{0}^{-2}=0.028$. The truncated expressions based on two-term expansions will be shown to describe the statistics of particle dispersion with errors of a few percent in important cases of turbulent flow.

\section{CONNECTION TO HAMILTONIAN DYNAMICS}

We shall concentrate on terms of leading order with respect to $C_{0}$ in the damping function. Dropping the second, third, and fourth terms on the right hand side of Eq. (17), we have

$$
\frac{d v_{i}^{\prime}}{d t^{\prime}}=-\frac{1}{2} C_{0} \varepsilon \lambda_{i j} v_{j}^{\prime}+a_{i}^{\prime H}+\left(C_{0} \varepsilon\right)^{1 / 2} w_{i}\left(t^{\prime}\right),
$$

where the homogeneous solution $a_{i}^{\prime H}$ has been retained; because of nonuniqueness, its value is yet unknown. Specifying the magnitude of $a_{i}^{\prime H}$ with respect to $C_{0}$ is the objective of 
the subsequent analysis. Suppose for the time being that $a_{i}^{\prime H}$ competes in the leading order formulation with respect to $C_{0}$ of the damping function. To express this explicitly, we write

$$
a_{i}^{\prime H}=C_{0} a_{i}^{\prime * H} .
$$

Furthermore, from the Langevin equation (19), it follows that fluctuations of velocity take place on time scales proportional to $C_{0}^{-1}$. Therefore we introduce the time $t^{*}$ defined by

$$
t^{*}=C_{0} t^{\prime},
$$

so that the Langevin equation (19) becomes

$$
\frac{d v_{i}^{\prime}}{d t^{*}}=-\frac{1}{2} \varepsilon \lambda_{i j} v_{j}^{\prime}+a_{i}^{\prime * H}+\varepsilon^{1 / 2} w_{i}\left(t^{*}\right) .
$$

A further simplification can be achieved by noting that the typical time scale of velocity fluctuations corresponds to $t^{*}=\mathcal{O}(1)$ or $t^{\prime}=\mathcal{O}\left(C_{0}^{-1}\right)$. Consider now fluid particles that are passively marked at time $t^{*}=t^{\prime}=0$ at position $\boldsymbol{x}=\boldsymbol{x}_{0}$. Each marked particle has a randomly chosen velocity at $t=0$ according to a probability distribution, which corresponds to the Gaussian Eulerian velocity distribution at the point of marking. Now, if $C_{0} \gg 1$, such particles will have moved hardly during a time $t^{\prime}=\mathcal{O}\left(C_{0}^{-1}\right)$. Considering the dynamics of such particles as described by Eq. (22), we can assume that $\varepsilon$ and $\lambda_{i j}$, which vary with space and time, will hardly have changed in magnitude and will be almost equal to their values at $t^{\prime}=0$ and $\boldsymbol{x}=\boldsymbol{x}_{0}$, i.e., $\varepsilon=\varepsilon_{0}$ and $\lambda_{i j}=\lambda_{i j, 0}$ in the leading order formulation with respect to $C_{0}$. Furthermore, we note that the energy dissipation rate $\varepsilon=\varepsilon_{0}$ is a measure for the rate in time by which energy flows from large energetic scales to the small dissipative scales: $\left(d / d t^{\prime}\right) H \sim \varepsilon_{0}$. On the much smaller time scale $t^{*}$, this energy change is small. Because $\Delta H \sim C_{0}^{-1} \varepsilon_{0} t^{*}$, the energy change becomes disregardingly small when $C_{0} \gg 1$ and $t^{*}=\mathcal{O}(1)$. The result is that the underlying dynamics of particle motion become those of a Hamiltonian system. The time scales of the velocity dynamics are large with respect to Kolmogorov's viscous time because $\operatorname{Re} \gg 1$ and small compared to the time at which energy is added or subtracted from the particle because $C_{0} \gg 1$. It corresponds to the inertial subrange of times, the area where the so-called parcel dynamics based on a Hamiltonian description of fluid flow are considered to be appropriate. ${ }^{27}$ The above analysis shows that for $C_{0} \gg 1$, a complete statistical process for particle velocities is established within such time range. The process represents a partial equilibrium which will be distorted for larger times in which case the coefficients in Eq. (22) will change. During the short times when partial equilibrium exists, $t^{*}=\mathcal{O}(1)$, the velocity process is equivalent to that of Hamiltonian systems considered in statistical physics. ${ }^{5-7}$ Onsager's reciprocity conditions must hold: the coefficient of the damping term must be symmetrical. It rules out the existence of the linear homogeneous solution given by Eq. (14), which is nonsymmetrical, i.e., $b_{k}=0$. Furthermore, as the statistical process of Hamiltonian nondissipative systems is entirely Gaussian exponentially correlated, nonlinear formulations such as those of Eq. (15) cannot exist either. Conclusion is that in the leading order formulation with respect to $C_{0}, a_{i}^{\prime * H}$ can be set equal to zero.
Nonuniqueness is thus deferred to a lower order formulation in $C_{0}$, i.e., $a_{i}^{\prime * H}=C_{0}^{-1} a_{i}^{\prime H}$, where $a_{i}^{\prime H}$ is of order unity with respect to $C_{0}$. The representation of the Langevin equation by Eq. (17) complies with this situation. The function $a_{i}^{\prime H}$ will be subjected to uncertainty which is reflected in the unknown constants in any of the solutions of Eq. (12) or Eq. (13). However, the magnitude of these constants will be such that the magnitude of $a_{i}^{\prime H}$ is comparable to that of $C_{0}^{-1}$ times the leading term in the damping function. This is a consequence of our finding that in the leading order formulation with respect to $C_{0}$, the dynamics of particle velocity are equivalent to those of nondissipative classical Hamiltonian systems. Thereby it is noted that as a result of our analysis no term in the damping function has been dropped. All that has been done is deriving a connection with Hamiltonian dynamics to establish the magnitude with respect to $C_{0}$ of the unknown homogeneous term in the damping function. This result appears to be most rewarding. It turns out that the obtained scaling of $a_{i}^{\prime H}$ results in contributions of only $\mathcal{O}\left(C_{0}^{-2}\right)$ in displacement statistics. The nonunique term is thus found to be of minor importance in the description of dispersion thus providing a way out to the nonuniqueness problem.

\section{DIFFUSION LIMIT}

Our aim is to construct an accurate statistical model for particle trajectories in turbulent flow. So far, we have arrived at a Langevin equation with damping function in which the contributions of the various terms are properly ordered in terms of their magnitudes with respect to $C_{0}$. To proceed further, we need to know how these terms contribute in the process of particle displacement. Particle displacements of appreciable magnitude take place on a much longer time scale than the time scale over which the particle velocities are correlated. The proper way to analyze particle displacement is to develop expressions from the presented Langevin equation, which hold for long times after marking. This is known as the diffusion limit. It will be presented below.

The Fokker-Planck equation for the time-dependent joint probability density $p=p\left(\boldsymbol{v}^{\prime}, \boldsymbol{x}^{\prime}, t^{\prime}\right)$ of particle velocity and particle position associated with fluctuation equations (17) and (18) can be formulated as ${ }^{28}$

$$
\begin{aligned}
\frac{\partial p}{\partial t^{\prime}}= & \frac{1}{2} \varepsilon C_{0}\left\{\frac{\partial}{\partial v_{i}^{\prime}}\left(\lambda_{i j} v_{j}^{\prime} p\right)+\frac{\partial^{2} p}{\partial v_{i}^{\prime} \partial v_{i}^{\prime}}\right\}-\frac{\partial}{\partial v_{i}^{\prime}}\left(\phi_{i} p\right) \\
& -\frac{\partial}{\partial x_{i}^{\prime}}\left(v_{i}^{\prime} p\right),
\end{aligned}
$$

where

$$
\phi_{i}=\frac{1}{2} \lambda_{j m} \frac{\partial \sigma_{m i}}{\partial t^{\prime}} v_{j}^{\prime}+\frac{\partial \sigma_{i k}}{\partial x_{k}^{\prime}}+\frac{1}{2} \lambda_{j n} \frac{\partial \sigma_{i j}}{\partial x_{m}^{\prime}}\left(v_{m}^{\prime} v_{n}^{\prime}-\sigma_{m n}\right)+a_{i}^{\prime H} .
$$

A well-known method to study the long-time behavior of solutions of the Fokker-Planck equation is homogenization. ${ }^{2}$ The basis of the method is a perturbation scheme which uses the property that for long times after marking concentrations become smooth and their spatial derivatives small. However, 
in the present version of the Fokker-Planck equation appropriate for turbulence, the method is not of much use. Spatial variations of Eulerian averages, in particular, the mean energy dissipation rate $\varepsilon$, are for general forms of turbulence large. The result is that higher order derivations do not become small as a result of homogenization. The only exception is the special case of (almost) homogeneous stationary turbulence. ${ }^{2}$ Another complicating factor in formulating a perturbation scheme appropriate for the diffusion limit is the absence of a dimensionless number in the Fokker-Planck equation. Just as for the Langevin equation discussed in Sec. IV, nondimensionalization of the Fokker-Planck equation (23) using $u_{*}$ and $L$ as characteristic values for velocity and length of the large scales does not yield a dimensionless number that can be used as perturbation parameter. In general, there exists no limit process by which the diffusion limit of inhomogeneous turbulence becomes exact. ${ }^{3}$ What rests is to develop a diffusion limit that is based on the smallness of $C_{0}^{-1}$. The procedure is similar to the method used by van Kampen to analyze Kramers' equation. ${ }^{4}$

The solution of Eq. (23) is written as an expansion in powers of $C_{0}^{-1}$,

$$
p=p_{0}+C_{0}^{-1} p_{1}+C_{0}^{-2} p_{2}+C_{0}^{-3} p_{3} .
$$

Equating terms of leading order in $C_{0}$ yields for $p_{0}$ the equation

$$
\frac{\partial}{\partial v_{i}^{\prime}}\left(\lambda_{i j} v_{j}^{\prime} p_{0}\right)+\frac{\partial^{2} p_{0}}{\partial v_{i}^{\prime} \partial v_{i}^{\prime}}=0,
$$

the solution of which is given by

$$
p_{0}=G_{0}\left(\boldsymbol{x}^{\prime}, t^{\prime}\right) p_{G}\left(\boldsymbol{v}^{\prime}\right),
$$

where $p_{G}=p_{G}\left(\boldsymbol{v}^{\prime}\right)$ is the Gaussian distribution given by Eq. (5). The function $G_{0}\left(x^{\prime}, t^{\prime}\right)$ describes the distribution of particle position or, equivalently, the mean concentration distribution of passively added admixture

$$
G_{0}\left(\boldsymbol{x}^{\prime}, t^{\prime}\right)=\int_{-\infty}^{+\infty} p_{0}\left(\boldsymbol{x}^{\prime}, \boldsymbol{v}^{\prime}, t^{\prime}\right) d \boldsymbol{v}^{\prime} .
$$

The equation that determines $G_{0}=G_{0}\left(\boldsymbol{x}^{\prime}, t^{\prime}\right)$ can be derived from the relationships which are obtained by substituting Eq. (25) into Eq. (23) and equating terms of lower order in $C_{0}^{-1}$. This yields for $p_{1}$ the equation

$$
\begin{aligned}
\frac{\partial}{\partial v_{i}^{\prime}} & \left(\lambda_{i j} v_{j}^{\prime} p_{1}\right)+\frac{\partial^{2} p_{1}}{\partial v_{i}^{\prime} \partial v_{i}^{\prime}} \\
& =2 \varepsilon^{-1}\left\{\frac{\partial}{\partial v_{i}^{\prime}}\left(\phi_{i} p_{0}\right)+\frac{\partial}{\partial x_{i}^{\prime}}\left(v_{i}^{\prime} p_{0}\right)+\frac{\partial p_{0}}{\partial t^{\prime}}\right\} .
\end{aligned}
$$

Now integrate this equation over the entire $\boldsymbol{v}^{\prime}$-domain. Because $p_{1}$ and its derivative can be assumed to decrease exponentially fast to zero as $\left|\boldsymbol{v}^{\prime}\right| \rightarrow \infty$, the integral of the right hand side must go to zero when $\left|\boldsymbol{v}^{\prime}\right| \rightarrow \infty$ as well. Noting that $\phi_{i} p_{0} \rightarrow 0$ exponentially fast as $\left|\boldsymbol{v}^{\prime}\right| \rightarrow \infty$ and that $\int_{-\infty}^{+\infty} v_{i}^{\prime} p_{0} d \boldsymbol{v}^{\prime}$ $=0$, we obtain the result that $\left(\partial / \partial t^{\prime}\right) G_{0}\left(\boldsymbol{x}^{\prime}, t^{\prime}\right)=0: G_{0}\left(\boldsymbol{x}^{\prime}, t^{\prime}\right)$ is required to be constant on the time scale $t^{\prime}=\mathcal{O}(1)$. It can only vary with time over a slower time scale. To account for this situation, we introduce the time

$$
t^{\prime \prime}=C_{0}^{-1} t^{\prime} .
$$

The two time scales of our problem now are the time scale $t^{\prime}=\mathcal{O}(1)$ over which the Eulerian statistical averages present as parameters in the Fokker-Planck equation vary and the slower time scale $t^{\prime \prime}=\mathcal{O}(1)$ over which particle dispersion takes place: $G_{0}\left(\boldsymbol{x}^{\prime}, t^{\prime}\right) \rightarrow G_{0}\left(\boldsymbol{x}^{\prime}, t^{\prime \prime}\right)$. Equation (29) is replaced by

$$
\begin{aligned}
\frac{\partial}{\partial v_{i}^{\prime}}( & \left.\lambda_{i j} v_{j}^{\prime} p_{1}\right)+\frac{\partial^{2} p_{1}}{\partial v_{i}^{\prime} \partial v_{i}^{\prime}} \\
= & 2 \varepsilon^{-1}\left\{G_{0}\left(\frac{\partial}{\partial v_{i}^{\prime}}\left(\phi_{i} p_{G}\right)+v_{i}^{\prime} \frac{\partial p_{G}}{\partial x_{i}^{\prime}}+\frac{\partial p_{G}}{\partial t^{\prime}}\right)\right. \\
& \left.+v_{i}^{\prime} p_{G} \frac{\partial G_{0}}{\partial x_{i}^{\prime}}\right\},
\end{aligned}
$$

where use was made of Eq. (27). From the well-mixed principle, it follows that

$$
\frac{\partial}{\partial v_{i}^{\prime}}\left(\phi_{i} p_{G}\right)+v_{i}^{\prime} \frac{\partial p_{G}}{\partial x_{i}^{\prime}}+\frac{\partial p_{G}}{\partial t^{\prime}}=0
$$

this relation is obtained upon substituting $a_{i}^{\prime}=-\frac{1}{2} C_{0} \varepsilon \lambda_{i j} v_{j}^{\prime}$ $+\phi_{i}$ and $p=p_{G}$ into Eq. (3). Implementing relation (32) into Eq. (31) yields

$$
\frac{\partial}{\partial v_{i}^{\prime}}\left(\lambda_{i j} v_{j}^{\prime} p_{1}\right)+\frac{\partial^{2} p_{1}}{\partial v_{i}^{\prime} \partial v_{i}^{\prime}}=2 \varepsilon^{-1} v_{i}^{\prime} p_{G} \frac{\partial G_{0}}{\partial x_{i}^{\prime}} .
$$

The solution is given by

$$
p_{1}=G_{1}\left(\boldsymbol{x}, t^{\prime \prime}\right) p_{G}-2 \varepsilon^{-1} \sigma_{n k} \frac{\partial G_{0}}{\partial x_{k}^{\prime}} v_{n}^{\prime} p_{G},
$$

where the second term is found as a particular solution of Eq. (33), while the first is the homogeneous solution.

The equation determining $p_{2}$ follows from the substitution of Eq. (25) into Eq. (23) and equating terms with appropriate powers of $C_{0}$. Noting the different contributions of the terms involving the time derivative because of the different time scales, we have

$$
\begin{aligned}
\frac{\partial}{\partial v_{i}^{\prime}} & \left(\lambda_{i j} v_{j}^{\prime} p_{2}\right)+\frac{\partial^{2} p_{2}}{\partial v_{i}^{\prime} \partial v_{i}^{\prime}} \\
& =2 \epsilon^{-1}\left\{\frac{\partial}{\partial v_{i}^{\prime}}\left(\phi_{i} p_{1}\right)+\frac{\partial}{\partial x_{i}^{\prime}}\left(v_{i}^{\prime} p_{1}\right)+\frac{\partial p_{1}}{\partial t^{\prime}}+p_{G} \frac{\partial G_{0}}{\partial t^{\prime \prime}}\right\} .
\end{aligned}
$$

Similar to the procedure subsequent to Eq. (29), we integrate Eq. (35) over the entire $\boldsymbol{v}^{\prime}$-domain. The integrals of the terms on the left hand side and of the first term on the right hand side can be assumed to go to zero as $\left|\boldsymbol{v}^{\prime}\right| \rightarrow \infty$. Furthermore, $\left(\partial / \partial t^{\prime}\right) \int_{-\infty}^{+\infty} p_{1} d \boldsymbol{v}^{\prime}=0$ so that

$$
\frac{\partial G_{0}}{\partial t^{\prime \prime}}+\frac{\partial}{\partial x_{i}^{\prime}}\left(\int_{-\infty}^{+\infty} v_{i}^{\prime} p_{1} d \boldsymbol{v}^{\prime}\right)=0,
$$

which upon substitution of solution (34) yields 


$$
\frac{\partial G_{0}}{\partial t^{\prime \prime}}=2 \frac{\partial}{\partial x_{i}^{\prime}}\left(\varepsilon^{-1} \sigma_{i n} \sigma_{n k} \frac{\partial G_{0}}{\partial x_{k}^{\prime}}\right) .
$$

Transforming to the nonmoving inertial coordinates $(\boldsymbol{x}, t)$ using Eqs. (4) and (30), this becomes

$$
\frac{\partial G_{0}}{\partial t}+u_{i}^{0} \frac{\partial G_{0}}{\partial x_{i}}=\frac{\partial}{\partial x_{i}}\left(D_{i k}^{0} \frac{\partial G_{0}}{\partial x_{k}}\right)
$$

where $D_{i k}^{0}$ is turbulent diffusivity defined by

$$
D_{i k}^{0}=2 C_{0}^{-1} \varepsilon^{-1} \sigma_{i n} \sigma_{n k} .
$$

The above result is the classical diffusion equation. It has been derived before ${ }^{2}$ but by applying methods of homogenization assuming almost homogeneous stationary turbulence. Furthermore, the damping function was taken linear in velocity, whereby its coefficients were supposed to be known, thus circumpassing the problem of nonuniqueness. The above result was obtained without such assumptions. It emerged as the leading term of an expansion in powers of $C_{0}^{-1}$. It holds for general forms of turbulence provided terms next to leading order in $C_{0}^{-1}$ can be disregarded. The terms in the Langevin equation which suffer from nonuniqueness are found not to contribute in the leading order formulation. This is a direct consequence of the earlier result (Sec. V) that in the leading order formulation with respect to $C_{0}^{-1}$, the dynamics of particle velocity can and have been assumed to resemble that of a Hamiltonian process. Nonuniqueness can only play a role in higher order formulations. Such formulations will be presented in Sec. VII.

\section{HIGHER ORDER DIFFUSION LIMIT}

Substituting Eq. (25) into Eq. (23) and equating terms of appropriate powers of $C_{0}^{-1}$, we have for $p_{3}$ the equation

$$
\begin{aligned}
& \frac{\partial}{\partial v_{i}^{\prime}}\left(\lambda_{i j} v_{j}^{\prime} p_{3}\right)+\frac{\partial^{2} p_{3}}{\partial v_{i}^{\prime} \partial v_{i}^{\prime}} \\
& \quad=2 \varepsilon^{-1}\left\{\frac{\partial}{\partial v_{i}^{\prime}}\left(\phi_{i} p_{2}\right)+\frac{\partial}{\partial x_{i}^{\prime}}\left(v_{i}^{\prime} p_{2}\right)+\frac{\partial p_{2}}{\partial t^{\prime}}+\frac{\partial p_{1}}{\partial t^{\prime \prime}}\right\} .
\end{aligned}
$$

Now, we integrate this equation with respect to $\boldsymbol{v}^{\prime}$ over its entire $\boldsymbol{v}^{\prime}$-domain. As before, the then obtained integrals of the terms on the left and the first on the right will vanish because of assumed exponential decay of probability densities as $\left|v^{\prime}\right| \rightarrow \infty$. Furthermore, from Eq. (35) one can derive a solution for $p_{2}$ of the form

$$
p_{2}=G_{2}\left(\boldsymbol{x}^{\prime}, t^{\prime \prime}\right) p_{G}+\kappa_{i} v_{i}^{\prime} p_{G}+\kappa_{m n}\left(v_{m}^{\prime} v_{n}^{\prime}-\sigma_{m n}\right) p_{G},
$$

where the first term on the right hand side is the homogeneous solution, while the second and third terms are particular solutions. Expressions for the coefficients $\kappa_{i}$ and $\kappa_{m n}$ are obtained through substitution into Eq. (35) and equating like terms. From solution (41), it is found that $\left(\partial / \partial t^{\prime}\right) \int_{-\infty}^{+\infty} p_{2} d \boldsymbol{v}^{\prime}$ $=0$ so that the integral of Eq. (40) becomes

$$
\frac{\partial}{\partial x_{i}^{\prime}} \int_{-\infty}^{+\infty} v_{i}^{\prime} p_{2} d \boldsymbol{v}^{\prime}+\frac{\partial G_{1}}{\partial t^{\prime \prime}}=0,
$$

where use was made of solution (34). To determine the value of the integral in Eq. (42), we multiply Eq. (35) by $v_{k}^{\prime}$ and integrate over the entire $\boldsymbol{v}^{\prime}$-domain. Applying partial integration, we obtain

$$
\begin{aligned}
-\lambda_{k j} \int_{-\infty}^{+\infty} v_{j}^{\prime} p_{2} d \boldsymbol{v}^{\prime} \\
=-2 \varepsilon^{-1}\left\{\int_{-\infty}^{+\infty} \phi_{k} p_{1} d \boldsymbol{v}^{\prime}-\frac{\partial}{\partial x_{i}^{\prime}} \int_{-\infty}^{+\infty} v_{k}^{\prime} v_{i}^{\prime} p_{1} d \boldsymbol{v}^{\prime}\right. \\
\left.-\frac{\partial}{\partial t^{\prime}} \int_{-\infty}^{+\infty} v_{k}^{\prime} p_{1} d \boldsymbol{v}^{\prime}\right\},
\end{aligned}
$$

where the integral of the last term has been set equal to zero because the Gaussian distribution is zero-mean. Upon substituting the solution for $p_{1}$ given by Eq. (34), the integrals on the right hand side of the above equation can be evaluated as follows:

$$
\frac{\partial}{\partial t^{\prime}} \int_{-\infty}^{+\infty} v_{k}^{\prime} p_{1} d \boldsymbol{v}^{\prime}=-2 \varepsilon^{-1} \frac{\partial G_{0}}{\partial x_{j}} \frac{\partial}{\partial t^{\prime}}\left(2 \varepsilon^{-1} \sigma_{n j} \sigma_{n k}\right)=0,
$$

where it is noted that by virtue of the expansion leading to the diffusion equation [cf. Eq. (37)], the parameter combination $\varepsilon^{-1} \sigma_{n j} \sigma_{n k}$ is only allowed to vary over the second "slow" time $t$ ". In practice, this condition seems to be amply satisfied. In wall-induced turbulence, $\varepsilon$ and $\sigma_{n j}$ hardly vary in the direction of the mean flow; in case of developed pipe and channel flow they are even constant. ${ }^{1}$ In decaying isotropic turbulence behind a grid, variance of velocity and mean energy dissipation rate decay, respectively, as $t^{-1}$ and $t^{-2}$ in the limit of large Reynolds number ${ }^{21}$ so that $\varepsilon^{-1} \sigma_{n j} \sigma_{j k}$ is constant with respect to time.

For the second integral on the right hand side of Eq. (43), we can write

$$
\frac{\partial}{\partial x_{i}^{\prime}} \int_{-\infty}^{+\infty} v_{k}^{\prime} v_{i}^{\prime} p_{1} d \boldsymbol{v}^{\prime}=\frac{\partial}{\partial x_{i}^{\prime}}\left(G_{1} \sigma_{k i}\right)
$$

where we used the property that the third moment of a zeromean Gaussian distribution is zero. Upon substituting Eqs. (24) and (34), the first integral on the right hand side of Eq. (43) becomes

$$
\begin{aligned}
\int_{-\infty}^{+\infty} \phi_{k} p_{1} d \boldsymbol{v}^{\prime}= & \frac{\partial \sigma_{k i}}{\partial x_{i}^{\prime}} G_{1}+G_{1} \int_{-\infty}^{+\infty} a_{k}^{\prime \mathrm{H}} p_{G} d \boldsymbol{v}^{\prime} \\
& -\lambda_{j m} \frac{\sigma_{m k}}{\partial t^{\prime}} \varepsilon^{-1} \sigma_{n l} \frac{\partial G_{0}}{\partial x_{l}^{\prime}} \sigma_{n j} \\
& -2 \varepsilon^{-1} \sigma_{n l} \frac{\partial G_{0}}{\partial x_{l}^{\prime}} \int_{-\infty}^{+\infty} a_{k}^{\prime \mathrm{H}} v_{n}^{\prime} p_{G} d \boldsymbol{v}^{\prime} .
\end{aligned}
$$

Now multiply Eq. (13) with $u_{j}^{\prime}$, integrate over the entire $\boldsymbol{u}^{\prime}$-domain, and apply partial integration

$$
\int_{-\infty}^{+\infty} a_{i}^{\prime H} p_{G} d \boldsymbol{u}^{\prime}=0
$$

Using this result in Eq. (46), substituting results (44)-(46) into Eq. (43), and implementing the resulting equation for $\int_{-\infty}^{+\infty} v_{i}^{\prime} p_{2} d \boldsymbol{v}^{\prime}$ into Eq. (42) yields 


$$
\begin{aligned}
\frac{\partial G_{1}}{\partial t^{\prime \prime}}= & 2 \frac{\partial}{\partial x_{i}^{\prime}}\left[\left(\varepsilon^{-1} \sigma_{k l} \sigma_{i k} \frac{\partial G_{1}}{\partial x_{l}^{\prime}}\right)+\varepsilon^{-2} \sigma_{i k} \sigma_{m l} \frac{\partial \sigma_{m k}}{\partial t^{\prime}} \frac{\partial G_{0}}{\partial x_{l}^{\prime}}\right. \\
& \left.+2 \varepsilon^{-2} \sigma_{n l} \sigma_{i k} \gamma_{k n} \frac{\partial G_{0}}{\partial x_{l}^{\prime}}\right],
\end{aligned}
$$

where the contribution of the term in the Langevin equation which suffers from nonuniqueness appears in the tensor

$$
\gamma_{k n}=\int_{-\infty}^{+\infty} a_{k}^{\prime H} v_{n}^{\prime} p_{G} d \boldsymbol{v}^{\prime}
$$

Converting to the fixed inertial frame including implementation of Eq. (30) gives

$$
\begin{aligned}
\frac{\partial G_{1}}{\partial t}+u_{i}^{0} \frac{\partial G_{1}}{\partial x_{i}}= & \frac{\partial}{\partial x_{i}}\left(D_{i k}^{0} \frac{\partial G_{1}}{\partial x_{k}}\right)+\frac{\partial}{\partial x_{i}}\left(D_{i k}^{\prime} \frac{\partial G_{0}}{\partial x_{k}}\right) \\
& +C_{0}^{-1} \frac{\partial}{\partial x_{i}}\left(d_{i k} \frac{\partial G_{0}}{\partial x_{k}}\right),
\end{aligned}
$$

where

$$
D_{i k}^{\prime}=2 C_{0}^{-1} \varepsilon^{-2} \sigma_{l i} \sigma_{k j} u_{n}^{0} \frac{\partial \sigma_{l j}}{\partial x_{n}},
$$

where because of stationarity in the fixed inertial frame we implemented $\left(\partial / \partial t^{\prime}\right) \sigma_{l j}=u_{n}^{0}\left(\partial / \partial x_{n}\right) \sigma_{l j}$. Furthermore,

$$
d_{i k}=2 \varepsilon^{-2} \sigma_{n k} \sigma_{i l} \gamma_{l n} .
$$

Now multiply Eq. (13) by $v_{n}^{\prime} v_{m}^{\prime}$ and integrate over the entire $\boldsymbol{v}^{\prime}$-domain. Partial integration then yields

$$
\int_{-\infty}^{+\infty} v_{m}^{\prime} a_{n}^{\prime H} p_{G} d \boldsymbol{v}+\int_{-\infty}^{+\infty} v_{n}^{\prime} a_{m}^{\prime H} p_{G} d \boldsymbol{v}=0
$$

and for $\gamma_{l n}$ defined by Eq. (49),

$$
\gamma_{l n}+\gamma_{n l}=0 \text {. }
$$

The tensors $\gamma_{l n}$ and $d_{i k}$ are thus antisymmetric. We can therefore write

$$
\frac{\partial}{\partial x_{i}}\left(d_{i k} \frac{\partial G_{0}}{\partial x_{k}}\right)=\frac{\partial d_{i k}}{\partial x_{i}} \frac{\partial G_{0}}{\partial x_{k}},
$$

and Eq. (50) becomes

$$
\begin{aligned}
\frac{\partial G_{1}}{\partial t}+u_{i}^{0} \frac{\partial G_{1}}{\partial x_{i}}= & C_{0}^{-1} \frac{\partial d_{i k}}{\partial x_{i}} \frac{\partial G_{0}}{\partial x_{k}}+\frac{\partial}{\partial x_{i}}\left(D_{i k}^{0} \frac{\partial G_{1}}{\partial x_{k}}\right) \\
& +\frac{\partial}{\partial x_{i}}\left(D_{i k}^{\prime} \frac{\partial G_{0}}{\partial x_{k}}\right) .
\end{aligned}
$$

The probability density of particle position or mean concentration of passive admixture up to and including contributions of $\mathcal{O}\left(C_{0}^{-1}\right)$ can be described as

$$
G=\int_{-\infty}^{+\infty}\left(p_{0}+C_{0}^{-1} p_{1}\right) d \boldsymbol{v}=G_{0}+C_{0}^{-1} G_{1} .
$$

Multiplying Eq. (56) by $C_{0}^{-1}$ and adding this equation to Eq. (38) yields

$$
\frac{\partial G}{\partial t}+\left(u_{i}^{0}-C_{0}^{-2} \frac{\partial d_{k i}}{\partial x_{k}}\right) \frac{\partial G}{\partial x_{i}}=\frac{\partial}{\partial x_{i}}\left(D_{i k} \frac{\partial G}{\partial x_{k}}\right),
$$

where

$$
D_{i k}=D_{i k}^{0}+C_{0}^{-1} D_{i k}^{\prime} .
$$

The term $d_{k i}$ is the term that suffers from nonuniqueness [cf. Eqs. (49) and (52)]. Because of antisymmetry of $d_{k i}$, the contribution involving nonuniqueness has shifted from a diffusivelike term [cf. Eq. (50)] to a convectivelike term [cf. Eq. (58)]. As convectivelike term it directly compares with convection by mean flow and is only of relative magnitude $C_{0}^{-2}$. This is beyond the accuracy of a description in powers up to and including $C_{0}^{-1}$ and can (and should) be disregarded. The diffusion equation in which the coefficients involve a relative error of $C_{0}^{-2}$ is thus given by

$$
\frac{\partial G}{\partial t}+u_{i}^{0} \frac{\partial G}{\partial x_{i}}=\frac{\partial}{\partial x_{i}}\left(D_{i k} \frac{\partial G}{\partial x_{k}}\right) \text {. }
$$

The effect of nonuniqueness is absent in this two-term representation of the diffusion equation. The terms in the damping function of the Langevin equation, which describe the effect of nonuniqueness, yield contributions in the coefficients of the diffusion equation, which are of relative magnitude $C_{0}^{-2}$ only. It provides a pragmatic solution to the nonuniqueness problem. If we accept an error of $\mathcal{O}\left(C_{0}^{-2}\right)$ in dispersion statistics, we can disregard the contribution of the nonunique term, i.e., $a_{i}^{\prime \mathrm{H}}$ in Eq. (17), altogether. In Sec. X, we shall indicate in some detail the errors caused by omitting the nonunique term.

Another remarkable result is the correction in the turbulent diffusion coefficient in case of changing Eulerian covariances of velocity in the direction of mean velocity. In case of isotropic decaying turbulence behind a grid, we have according to the von Kármán-Howarth equation $u_{n}^{0}\left(\partial / \partial x_{n}\right) \sigma_{l j}$ $=-\frac{2}{3} \varepsilon \delta_{l j}$, which yields for the diffusivity [cf. Eqs. (39), (51), and (59)], the expression $D_{i k}=D_{i k}^{0}\left(1-\frac{2}{3} C_{0}^{-1}\right)$. Interestingly, there exists also an exact result for the diffusivity in decaying isotropic Gaussian turbulence: $D_{i k}=D_{i k}^{0}\left(1+\frac{2}{3} C_{0}^{-1}\right)^{-1}$ (see Appendix). It shows that our result is equal to the two-term expansion of the exact result, as should be. Furthermore, for $C_{0}=6$ the two-term expansion involves an error of only $1.2 \%$ compared to the exact result. It shows the effectiveness of the perturbation scheme when including terms of leading order and next to leading order in $C_{0}^{-1}$.

\section{LIGHT NON-GAUSSIANITY}

Results derived so far were based on the assumption that fixed-point Eulerian velocity statistics are Gaussian. Experimental observations tend to indicate some deviations of Gaussianity. This is apparent in values of skewness and kurtosis reaching in certain cases values of about 0.3. At the same time, we have found that statistics of Lagrangian-based particle velocities start to deviate from zero-mean Gaussian distributions when it concerns the contribution of terms of $\mathcal{O}\left(C_{0}^{-1}\right)$ [cf. Eq. (34)]. A rather logical approach is to assume that also fixed-point Eulerian velocities are zero-mean 
Gaussian in the leading order formulation with respect to $C_{0}$ but deviate from the Gaussian distribution in lower order formulations with respect to $C_{0}$,

$$
p_{E}\left(\boldsymbol{u}^{\prime}\right)=p_{G}\left(\boldsymbol{u}^{\prime}\right)+C_{0}^{-1} f_{c}\left(\boldsymbol{u}^{\prime}\right) p_{G}\left(\boldsymbol{u}^{\prime}\right),
$$

where $f_{c}\left(\boldsymbol{u}^{\prime}\right)$ describes the deviation from Gaussianity. As the first term in expansion (61) covers all probability, mean values and covariances, we have the property that

$$
\begin{aligned}
\int_{-\infty}^{+\infty} f_{c}\left(\boldsymbol{u}^{\prime}\right) p_{G}\left(\boldsymbol{u}^{\prime}\right) d \boldsymbol{u}^{\prime} & =\int_{-\infty}^{+\infty} u_{i}^{\prime} f_{c}\left(\boldsymbol{u}^{\prime}\right) p_{G}\left(\boldsymbol{u}^{\prime}\right) d \boldsymbol{u}^{\prime} \\
& =\int_{-\infty}^{+\infty} u_{m}^{\prime} u_{n}^{\prime} f_{c}\left(\boldsymbol{u}^{\prime}\right) p_{G}\left(\boldsymbol{u}^{\prime}\right) d \boldsymbol{u}^{\prime}=0
\end{aligned}
$$

For the damping function $a_{i}^{\prime}$ in the Langevin equation, we now can write

$$
a_{i}^{\prime}=-\frac{1}{2} C_{0} \varepsilon \lambda_{i j} v_{j}^{\prime}+\phi_{i}+\frac{1}{2} \varepsilon \frac{\partial f_{c}}{\partial v_{i}},
$$

where as before $\phi_{i}$ is described by Eq. (24). The above result is obtained through application of the well-mixed condition, i.e., substitution of Eq. (61) into Eq. (3) and solving for $a_{i}^{\prime}$ in the manner of Sec. III. The last term on the right hand side of Eq. (63) describes the effect of deviations by nonGaussianity through $f_{c}=f_{c}\left(\boldsymbol{v}^{\prime}\right)$. It is obtained as an additional particular solution for $a_{i}^{\prime}$.

When repeating the solution procedure of the diffusion limit for the case of light non-Gaussianity we have to replace $\phi_{i}$ by $\phi_{i}+\frac{1}{2} \varepsilon\left(\partial f_{c} / \partial v_{i}^{\prime}\right)$ everywhere in the formulas of Secs. VI and VII. The solution for the leading term in the expansion of $p$ is not affected by this replacement. Solution (27) still holds. The solution for $p_{1}$, however, changes as is apparent from the presence of the term involving $\phi_{i}$ on the right hand side of Eqs. (29) and (31). Replacing $\phi_{i}$ by $\phi_{i}$ $+\frac{1}{2} \varepsilon\left(\partial f_{c} / \partial v_{i}^{\prime}\right)$, the new version of Eq. (33) becomes

$$
\begin{aligned}
& \frac{\partial}{\partial v_{i}^{\prime}}\left(\lambda_{i j} v_{j}^{\prime} p_{1}\right)+\frac{\partial^{2} p_{1}}{\partial v_{i}^{\prime} \partial v_{i}^{\prime}} \\
& \quad=2 \varepsilon^{-1} v_{i}^{\prime} p_{G} \frac{\partial G_{0}}{\partial x_{i}^{\prime}}+G_{0} \frac{\partial}{\partial v_{i}^{\prime}}\left(p_{G} \frac{\partial f_{c}}{\partial v_{i}^{\prime}}\right),
\end{aligned}
$$

the solution of which is given by

$$
p_{1}=G_{1}\left(\boldsymbol{x}, t^{\prime \prime}\right) p_{G}+G_{0}\left(\boldsymbol{x}, t^{\prime \prime}\right) f_{c} p_{G}-2 \varepsilon^{-1} \sigma_{n k} \frac{\partial G_{0}}{\partial x_{k}^{\prime}} v_{n}^{\prime} p_{G},
$$

where the second term that is absent in solution (34) describes the effect of light non-Gaussianity. Equation (36) remains unaffected by a change in $\phi_{i}$. Substituting solution (65) into this equation yields again Eq. (37); because of property (62), the second term in solution (65) yields no contribution in the integral of Eq. (36). Conclusion is that in the leading order formulation the diffusion limit is unaffected by non-Gaussianity of fixed-point Eulerian velocity statistics of relative magnitude $C_{0}^{-1}$ [cf. Eq. (61)].

Assessing the effect of light non-Gaussianity on the higher order terms in the diffusion equation requires more extensive analysis. As $\phi_{i}$ changes in Eq. (40), solution (41) will change as well. However, this change does not affect result (42). In fact, we can start our analysis from Eq. (43) in which two sorts of additional terms arise. First of all, the solution for $p_{1}$ contains an extra term, the term involving $f_{c}$ [cf. Eq. (65)], which yields new contributions to the integrals of Eq. (43). Second, the term containing $\phi_{k}$ changes and has to be extended with the term $\frac{1}{2} \varepsilon\left(\partial / \partial v_{k}^{\prime}\right) f_{c}$. Let us first consider the contribution of the extra term in the solution for $p_{1}$. It yields in the second and third integrals on the right hand side of Eq. (43) contributions such as

$$
\int_{-\infty}^{+\infty} v_{k}^{\prime} v_{i}^{\prime} f_{c} p_{G} d \boldsymbol{v}^{\prime}, \quad \int_{-\infty}^{+\infty} v_{k}^{\prime} f_{c} p_{G} d \boldsymbol{v}^{\prime},
$$

which are equal to zero because of property (62). The extra contributions in the first integral on the right hand side of Eq. (43) are those involving the product of $\phi_{k}$, as given by the right hand side of Eq. (24) with the new contribution in $p_{1}$. Because of property (62), all such contributions are zero except for that due to $a_{k}^{\prime H}$ in Eq. (24). It yields the contribution to be added to the integrals on the right hand side of Eq. (43),

$$
-2 \varepsilon^{-1} G_{0} \int_{-\infty}^{+\infty} a_{k}^{\prime \mathrm{H}} f_{c} p_{G} d \boldsymbol{v}^{\prime}
$$

The last extra contribution arises because $\phi_{k}$ in the first integral of Eq. (43) has to be extended by $\frac{1}{2} \varepsilon\left(\partial / \partial v_{k}^{\prime}\right) f_{c}$ so that we obtain the extra integral

$$
-\int_{-\infty}^{+\infty} \frac{\partial f_{c}}{\partial v_{k}^{\prime}} p_{1} d \boldsymbol{v}^{\prime}
$$

Here, $p_{1}$ contains three terms [cf. Eq. (65)]. The contributions of the first and last of these terms in the integral, however, are zero. This follows from partial integration and noting that $\left(\partial / \partial v_{k}^{\prime}\right) p_{G}=-\lambda_{k i} v_{i}^{\prime} p_{G}$,

$$
\begin{aligned}
\int_{-\infty}^{+\infty} \frac{\partial f_{c}}{\partial v_{k}^{\prime}} p_{G} d \boldsymbol{v}^{\prime} & =-\int_{-\infty}^{+\infty} \frac{\partial p_{G}}{\partial v_{k}^{\prime}} f_{c} d \boldsymbol{v}^{\prime} \\
& =\lambda_{k i} \int_{-\infty}^{+\infty} v_{i}^{\prime} f_{c} p_{G} d \boldsymbol{v}^{\prime}=0, \\
\int_{-\infty}^{+\infty} \frac{\partial f_{c}}{\partial v_{k}^{\prime}} v_{n}^{\prime} p_{G} d \boldsymbol{v}^{\prime} & =-\int_{-\infty}^{+\infty} f_{c}\left(v_{n}^{\prime} \frac{\partial p_{G}}{\partial v_{k}^{\prime}}+\delta_{n k} p_{G}\right) d \boldsymbol{v}^{\prime} \\
& =+\int_{-\infty}^{+\infty} f_{c}\left(\lambda_{k i} v_{i}^{\prime} v_{n}^{\prime} p_{G}-\delta_{n k} p_{G}\right) d \boldsymbol{v}^{\prime}=0,
\end{aligned}
$$

where again use was made of property (62). The only contribution that remains is that of the second term in the solution of $p_{1}$. It is

$$
-G_{0} \int_{-\infty}^{+\infty} f_{c} \frac{\partial f_{c}}{\partial v_{k}^{\prime}} p_{G} d \boldsymbol{v}^{\prime}
$$

Conclusion is that in case of light non-Gaussianity, two contributions have to be added to the right hand side of Eq. (43), viz., contributions (67) and (71). However, as follows from 
the procedure leading to the diffusion equation upon substituting Eq. (43) into Eq. (42), these contributions involve first order derivatives with respect to $\boldsymbol{x}^{\prime}$ or $\boldsymbol{x}$. Hence, they result in corrections in the convective terms of the diffusion equation only. More specifically, they result in

$$
+C_{0}^{-2} \frac{\partial}{\partial x_{i}}\left(e_{i} G\right)
$$

where

$$
e_{i}=\sigma_{i k} \int_{-\infty}^{+\infty}\left(2 \varepsilon^{-1} a_{k}^{\prime H}+\frac{\partial f_{c}}{\partial v_{k}^{\prime}}\right) f_{c} p_{G} d \boldsymbol{v}^{\prime}
$$

to be added to the left hand side of Eq. (58). As the presented diffusion equation is accurate up to and including terms of $\mathcal{O}\left(C_{0}^{-1}\right)$, this contribution has to be abandoned. Light nonGaussianity of Eulerian fixed-point velocity statistics does not affect the two-term description of the diffusion limit. The underlying reason is that zero, first, and second order moments are not affected by non-Gaussianity [cf. Eq. (62)]. It is because of this property that potential first order corrections on diffusivity were nullified in the previous mathematical analysis.

\section{SUMMARY: DIFFUSION AND LANGEVIN EQUATION OF TURBULENT FLOW}

The analysis of Secs. VI-VIII has resulted in the diffusion equation, which describes the mean concentration of passive admixture or the probability density of the position of a marked fluid particle in turbulent flow. The equation is given by

$$
\frac{\partial G}{\partial t}+u_{i}^{0}(\boldsymbol{x}) \frac{\partial G}{\partial x_{i}}=\frac{\partial}{\partial x_{i}}\left(D_{i j}(x) \frac{\partial G}{\partial x_{j}}\right),
$$

where the symmetric diffusion constant $D_{i j}(\boldsymbol{x})$ is defined by

$$
\begin{aligned}
D_{i j}(\boldsymbol{x})= & 2 C_{0}^{-1} \varepsilon^{-1}(\boldsymbol{x}) \sigma_{i l}(\boldsymbol{x}) \sigma_{l j}(\boldsymbol{x}) \\
& +2 C_{0}^{-2} \varepsilon^{-2}(\boldsymbol{x}) \sigma_{l i}(\boldsymbol{x}) \sigma_{j k}(\boldsymbol{x}) u_{n}^{0}(\boldsymbol{x}) \frac{\partial \sigma_{l k}(\boldsymbol{x})}{\partial x_{n}} .
\end{aligned}
$$

The equation has been obtained through a perturbation expansion based on powers of the inverse Kolmogorov constant $C_{0}^{-1}$ which was truncated for terms of $\mathcal{O}\left(C_{0}^{-2}\right)$ and smaller. The equation holds for turbulent fluid flow in which fixed-point Eulerian fluid velocities are not far from Gaussian, to $\mathcal{O}\left(C_{0}^{-1}\right)$. While the equation has been derived for stationary turbulent flow in the fixed $(\boldsymbol{x}, t)$ frame, it can be applied to nonstationary turbulence in the fixed frame upon taking account of time-dependency of Eulerian averages in Eqs. (74) and (75) and replacing $u_{n}^{0}(\boldsymbol{x})\left(\partial / \partial x_{n}\right) \sigma_{i k}(\boldsymbol{x})$ by $(\partial / \partial t) \sigma_{i k}(\boldsymbol{x}, t)+u_{n}^{0}(\boldsymbol{x}, t)\left(\partial / \partial x_{n}\right) \sigma_{i k}(\boldsymbol{x}, t)$ in the expression for the turbulent diffusion coefficient.

Having specified the diffusion equation for turbulent particle dispersion we can return to the problem of specifying the damping function in the Langevin equation. There are two sorts of terms in the damping function whose specification is unknown: the terms that involve nonuniqueness [cf. $a_{i}^{\prime H}$ in Eq. (17)] and the terms that address slight deviations for Gaussianity of fixed-point Eulerian velocity [cf. $f_{c}$ in Eq.
(63)]. The analysis of Secs. VI-VIII has shown that these terms produce only corrections of relative magnitude $\mathcal{O}\left(C_{0}^{-2}\right)$ in the coefficients of the diffusion equation. It implies that when allowing for relative errors of $\mathcal{O}\left(C_{0}^{-2}\right)$ in dispersion calculations, the contributions of terms related to nonuniqueness and slight non-Gaussianity can be disregarded. Furthermore, also the zero-mean quadratic term in Eq. (17), which is associated with covariance inhomogeneity, i.e., $\frac{1}{2} \lambda_{j n}\left(\partial / \partial x_{m}\right) \sigma_{i j}\left(v_{m}^{\prime} v_{n}^{\prime}-\sigma_{m n}\right)$ can be dropped. As follows from the derivation of the diffusion equation, its contribution in the coefficients of the diffusion equation is $\mathcal{O}\left(C_{0}^{-2}\right)$ at most. The Langevin equation accurate in dispersion up to and including $\mathcal{O}\left(C_{0}^{-1}\right)$ is now given by

$$
\begin{aligned}
\frac{d v_{i}^{\prime}(t)}{d t}= & -\frac{1}{2} C_{0} \varepsilon(\boldsymbol{x}) \lambda_{i j}(\boldsymbol{x}) v_{j}^{\prime}(t) \\
& +\frac{1}{2} \lambda_{j m}(\boldsymbol{x}) u_{n}^{0}(\boldsymbol{x}) \frac{\partial \sigma_{m i}(\boldsymbol{x})}{\partial x_{n}} v_{j}^{\prime}(t)+\frac{\partial \sigma_{i n}}{\partial x_{n}} \\
& +\left[C_{0} \varepsilon(\boldsymbol{x})\right]^{1 / 2} w_{i}(t),
\end{aligned}
$$

where particle position follows from

$$
\frac{d x_{i}(t)}{d t}=u_{i}^{0}(\boldsymbol{x})+v_{i}^{\prime}(t) .
$$

As for the diffusion equation, the above equations have been derived for stationary turbulent flow in the fixed $(\boldsymbol{x}, t)$ frame. They can be applied to nonstationary turbulence in the fixed frame upon taking into account time-dependency of Eulerian-based averages and replacing $u_{n}^{0}(\boldsymbol{x})\left(\partial / \partial x_{n}\right) \sigma_{m i}(\boldsymbol{x})$ by $(\partial / \partial t) \sigma_{m i}(\boldsymbol{x}, t)+u_{n}^{0}(\boldsymbol{x}, t)\left(\partial / \partial x_{n}\right) \sigma_{m i}(\boldsymbol{x}, t)$ in the second term on the right hand side of Eq. (76).

The above presented diffusion equation and Langevin equation become equal to the previously presented equations for wall-induced turbulence ${ }^{29}$ upon implementing the assumption of Eulerian velocity statistics, which are constant in time and in the direction of the mean flow. In that case, the terms related to $u_{n}^{0}(\boldsymbol{x})$ in Eqs. (75) and (76) vanish. Furthermore, it is noted that the damping term in the Langevin equation is linear in velocity except from the drift term $\left(\partial / \partial x_{n}\right) \sigma_{i n}$, which contributes under inhomogeneous conditions. The presented Langevin equation is equal to a previously presented description of Thomson ${ }^{12,13}$ except from the zero-mean quadratic term $\frac{1}{2} \lambda_{j n}\left(\partial / \partial x_{m}\right) \sigma_{i j}\left(v_{m}^{\prime} v_{n}^{\prime}-\sigma_{m n}\right)$. As mentioned before, this term (as several other terms) can be (and have to be) dropped in any description that pertains to an accuracy of terms up to and including $\mathcal{O}\left(C_{0}^{-1}\right)$. The Thomson model is the result of selecting a symmetric form for the linear part of the damping term out of many other possible linear forms satisfying the well-mixed criterion. The same is done in selecting a quadratic form out of many other forms satisfying the well-mixed criterion. The selected descriptions comply with the assumed Gaussian fixed-point Eulerian velocity distribution. In another proposal, which is attributed to Borgas, ${ }^{12,13}$ the linear part of the damping term is antisymmetric. A linear description for $a_{i}^{\prime H}$ is postulated, which is of leading order in $C_{0}$ and which meets the wellmixed criterion. ${ }^{12,13}$ The different proposals made for the Langevin equation reflect the uncertainty caused by the prob- 
lem of nonuniqueness. The present analysis offers a way out to this problem. It shows that the terms, which cause uncertainty, when properly ordered in accordance with the outcome of the connection with Hamiltonian dynamics, yield contributions in dispersion which are only $\mathcal{O}\left(C_{0}^{-2}\right)$. The presented Langevin equation is the simplest possible version, which is unique and correct up to an error of $\mathcal{O}\left(C_{0}^{-2}\right)$ in dispersion. The diffusion equation follows from the longtime limit of the Langevin equation. Also, this equation is unique and accurate up to $\mathcal{O}\left(C_{0}^{-2}\right)$. The validity of the presented Langevin and diffusion equations is not restricted to a purely Gaussian fixed-point Eulerian velocity field. They hold for Eulerian velocity statistics which are non-Gaussian to a degree of $\mathcal{O}\left(C_{0}^{-1}\right)$. In Sec. X, we shall quantify in some detail the magnitude of the disregarded terms and their impact on accuracy.

\section{ACCURACY OF RESULTS}

The formulations of diffusion equation (74) and Langevin equation (76) are the result of perturbation expansions based on the inverse of the Kolmogorov constant $C_{0}$. The expansions have been truncated for terms which contribute to $\mathcal{O}\left(C_{0}^{-2}\right)$ in dispersion statistics. The errors caused by these truncations have different origins: (a) errors due to disregarding the nonunique terms in the Langevin equation; (b) errors related to the diffusion approximation; (c) errors associated with the description for decaying turbulence; and (d) errors due to non-Gaussianity of the Eulerian velocity field. Each of these errors will be specified in some detail below.

\section{A. Nonuniqueness}

By establishing a connection to Hamiltonian dynamics (Sec. V), we were able to show that the nonunique terms in the damping function of the Langevin equation are $\mathcal{O}\left(C_{0}^{-1}\right)$ compared to the terms of leading order. Invoking the diffusion approximation (Secs. VI and VII), it was found that the nonunique terms yield contributions of $\mathcal{O}\left(C_{0}^{-2}\right)$ in dispersion statistics. However, the analysis presented did not provide a clue to the precise value of these terms and the resulting error when dropping them. For that purpose, we shall resort to results of DNS and measurements obtained for limited but important cases of turbulent flow.

As shown in a previous article, ${ }^{29}$ a distinct property of the nonunique term described by Eq. (14) is that it causes antisymmetry in the cross-correlation functions of fluid particle velocity: $\overline{v_{i}^{\prime}(t) v_{j}^{\prime}(0)} \neq \overline{v_{j}^{\prime}(t) v_{i}^{\prime}(0)}$ for $i \neq j$. Here the overbar denotes Lagrangian averaging, viz., the average value of many realizations at some fixed moment in time $t$ assuming that all fluid particles start from the same fixed position at $t=0$ and that the particles are passively marked, i.e., they have randomly chosen values in accordance with the Eulerian velocity distribution at the point of marking. There exist data on cross-correlation functions of particle velocities obtained from measurements and DNS of wall-induced turbulence. ${ }^{30}$ With these data, one can determine the magnitude of the nonunique term, i.e., the value of the coefficients $b_{k}$ in Eq. (14), and subsequently its effect on dispersion statistics. ${ }^{29}$ It provides a possibility to quantify the accuracy

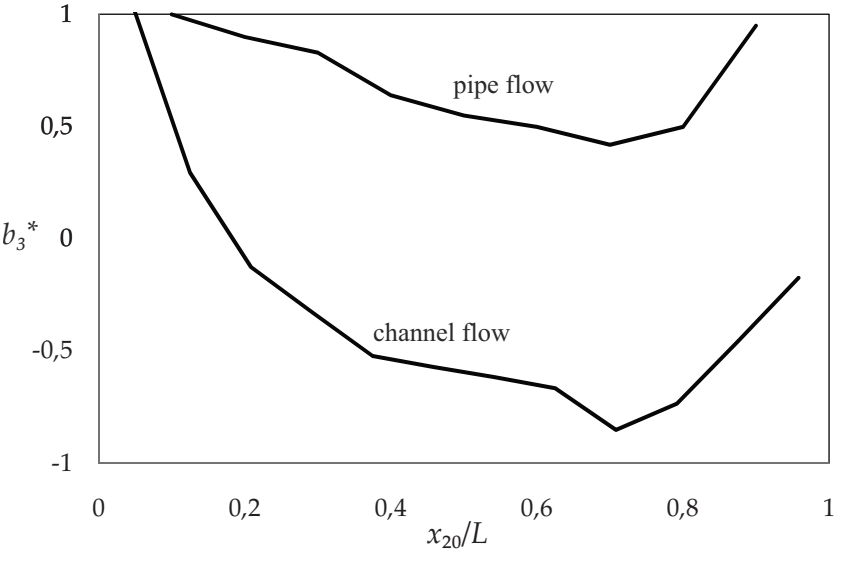

FIG. 1. Coefficient of asymmetry $b_{3}^{*}$ inferred from measurements in pipe flow and DNS of channel flow vs distance from the wall $x_{20}$ normalized with external length $L$ being the pipe radius or half-channel height, respectively. Results of measurements of pipe flow were provided by Walpot and results of DNS of channel flow by Kuerten.

of the present models [cf. Eqs. (74) and (76)] in which the contribution of the nonunique term has been disregarded.

In case of wall-induced turbulence, two out of three coefficients $b_{k}$ are zero. Velocity fluctuations in the direction perpendicular to the wall denoted by $x_{2}$ and in the direction of the mean flow parallel to the wall denoted by $x_{1}$ are uncorrelated with those in the $x_{3}$-direction. In other words, $a_{i}^{\prime H I}$ as described by Eq. (14), is not allowed to depend on $u_{3}^{\prime}$ when $i=1$ and $i=2$. This requires $b_{1}=b_{2}=0$. Now adding the remaining part of the nonunique term to the right hand side of the Langevin equation (76) and noting that in wallinduced turbulence Eulerian statistical averages only vary in $x_{2}$-direction, we have

$$
\begin{aligned}
\frac{d v_{i}^{\prime}}{d t}= & -\frac{1}{2} \varepsilon\left(x_{2}\right)\left[C_{0} \lambda_{i j}\left(x_{2}\right)+b_{3}^{*} \gamma_{i j}\left(x_{2}\right)\right] v_{j}(t)+\frac{d \sigma_{i 2}}{d x_{2}} \\
& +\left[C_{0} \varepsilon\left(x_{2}\right)\right]^{1 / 2} w_{i}(t),
\end{aligned}
$$

where $\lambda_{i j}\left(x_{2}\right)$ and $\gamma_{i j}\left(x_{2}\right)=\sigma_{i l} \varepsilon_{3 l j} d^{-1}$ are given by

$$
\begin{aligned}
& \lambda_{i j}=\left(\begin{array}{ccc}
d^{-1} \sigma_{22} & -d^{-1} \sigma_{12} & 0 \\
-d^{-1} \sigma_{12} & d^{-1} \sigma_{11} & 0 \\
0 & 0 & \sigma_{33}^{-1}
\end{array}\right), \\
& \gamma_{i j}=\left(\begin{array}{ccc}
-d^{-1} \sigma_{12} & d^{-1} \sigma_{11} & 0 \\
-d^{-1} \sigma_{22} & d^{-1} \sigma_{12} & 0 \\
0 & 0 & 0
\end{array}\right), \\
& d=\sigma_{11} \sigma_{22}-\sigma_{12}^{2}
\end{aligned}
$$

because $\sigma_{13}=\sigma_{23}=0$. In Eq. (78), $b_{3}^{*}$ is the dimensionless and normalized representation of $b_{3}$, which is related to $b_{3}$ by $b_{3}^{*}=2 b_{3} d \varepsilon^{-1}$. The magnitude of $b_{3}^{*}$ can now be determined by matching the differences between the cross-correlations $\overline{v_{1}^{\prime}(t) v_{2}^{\prime}(0)}$ and $\overline{v_{2}^{\prime}(t) v_{1}^{\prime}(0)}$ obtained from simulations based on Eq. (78) with those obtained from measurements and DNS. ${ }^{29}$ Results for $b_{3}^{*}$ thus obtained are presented in Fig. 1. Values shown for $b_{3}^{*}$ were derived from measurements using threedimensional particle tracking velocimetry applied to turbu- 

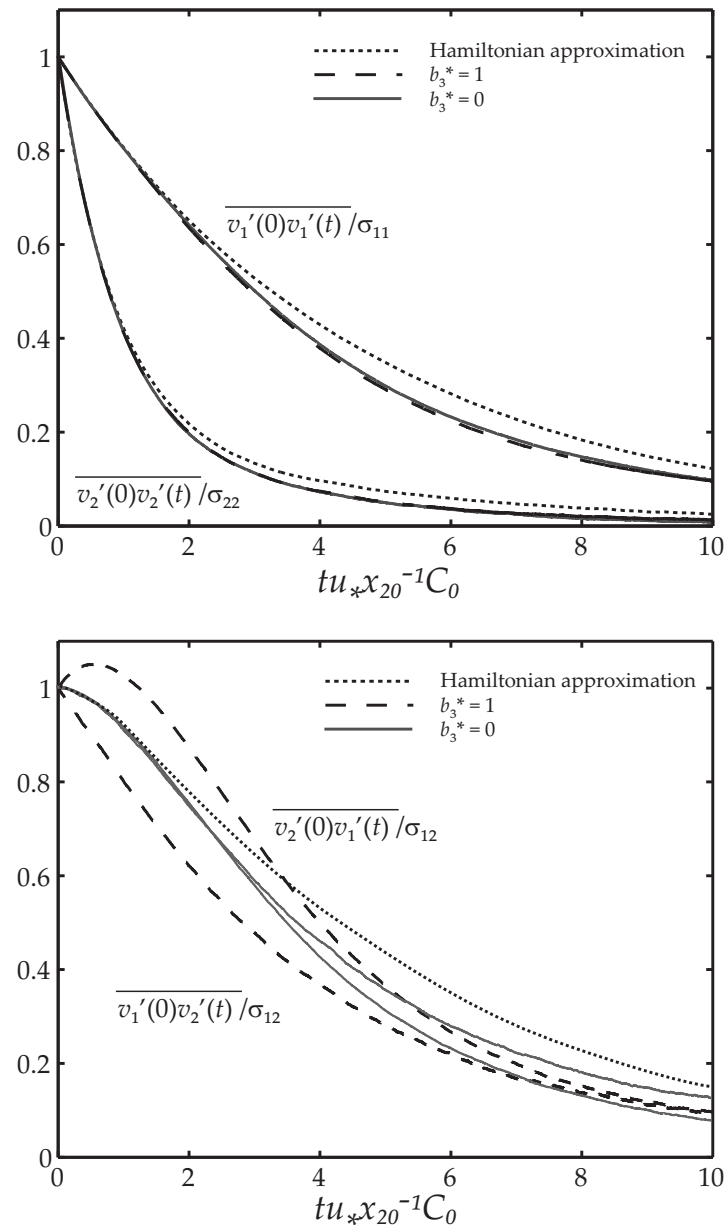

FIG. 2. Lagrangian velocity correlations for the log-layer of wall-induced turbulence obtained by $10^{6}$ simulations of the Langevin equation.

lent flow in a pipe. ${ }^{30}$ The Reynolds number based on pipe radius and shear velocity was 320 . Somewhat lower values for $b_{3}^{*}$ were obtained from DNS applied to the same configuration. Also shown in Fig. 1 are recent results of DNS applied to two-dimensional channel flow. ${ }^{31}$ The Reynolds number based on half-channel height and shear velocity was 590 . It is seen that $\left|b_{3}^{*}\right|$ is less than unity for the largest part of the cross-sectional area of the configurations.

The effect of the nonunique antisymmetric term on velocity correlation functions has been shown in Fig. 2. Results apply to the log-layer of wall-induced turbulence. In the loglayer, one can describe $\varepsilon\left(x_{2}\right)$ by the analytical expression ${ }^{26}$

$$
\varepsilon=\kappa^{-1} u_{*} x_{2}^{-1},
$$

where $\kappa$ is the von Kármán constant $(\kappa \approx 0.4)$ and $u_{*}$ is the shear velocity: $u_{*}=\sqrt{\tau_{0} / \rho}$, where $\tau_{0}$ is the mean shear stress at the wall and $\rho$ is the fluid density. Furthermore, in the log-layer one can assume $\sigma_{i j}$ to be constant with respect to $x_{2}$. Appropriate values are

$$
\sigma_{11}=5.67 u_{*}^{2}, \quad \sigma_{22}=1.32 u_{*}^{2}, \quad \sigma_{12}=-u_{*}^{2} .
$$

These values are in accordance with the measured values in high-Reynolds wall-induced turbulence ${ }^{32-34}$ and results of DNS. ${ }^{35}$ Shown in Fig. 2 are the results for $C_{0}=6$ considering three cases: (i) simulations based on the model described by
Eqs. (78)-(81) with $b_{3}^{*}=1$; (ii) simulations based on the same model but with $b_{3}^{*}=0$; and (iii) simulations based on Eqs. (78)-(81) but with $\varepsilon\left(x_{2}\right)$ kept constant and equal to its value at distance $x_{2}=x_{20}$, which is the point where particles are passively marked at $t=0: \varepsilon\left(x_{2}\right)=\kappa^{-1} u_{*} x_{20}^{-1}$. Furthermore, in case (iii) $b_{3}^{*}=0$. In cases (i) and (ii), particles were assumed to reflect at the wall $x_{2}=0$, while in case (iii) such a wall is absent. Case (i) in which asymmetry is fully taken into account is the reference case. Case (ii) is the prediction according to the present model. Case (iii) includes the approximation of local inhomogeneity which makes the model equivalent to the models of statistical physics based on Hamiltonian dynamics at microscopic level. It is seen from Fig. 2 that the results based on the Hamiltonian-like model, the present symmetric model and the antisymmetric reference model deviate somewhat from each other. However, deviations are limited. Results shown indicate that a value of $C_{0}=6$ results in stochastic behavior which is not so far from the "Hamiltonian limit." Mutual differences become somewhat larger when $C_{0}=5$ but are still limited. ${ }^{29}$

Disregarding the nonunique term in the damping function leads to errors of $\mathcal{O}\left(C_{0}^{-1}\right)$ in velocity statistics such as the velocity correlation functions shown in Fig. 2. On the other hand, our analysis has shown that the error caused by disregarding the nonunique term becomes only $\mathcal{O}\left(C_{0}^{-2}\right)$ when it concerns displacement statistics. This is confirmed by the results of simulations based on Eqs. (78)-(81). The statistics of particle displacement normal to the wall are hardly different when considering $b_{3}^{*}=0$ and $b_{3}^{*}=1{ }^{29}$ Even more convincing is the analytical expression for the correction on the diffusion constant in the diffusion equation of wall-induced turbulence. ${ }^{29}$ The antisymmetric term $b_{3}^{*} \gamma_{i j}\left(x_{2}\right)$ in Eq. (78) leads to a correction of the diffusion coefficient by the factor $1+b_{3}^{* 2} C_{0}^{-2}$. This result applies to the whole cross section of wall-induced turbulence and is thus not limited to the loglayer only. The correction factor amounts to a change of diffusivity of at most $2.8 \%$ for $C_{0}=6$ and $\left|b_{3}^{*}\right| \leq 1$.

The conclusions with regard to the magnitude of $b_{3}^{*}$ and its effect on statistics are also confirmed by the DNS results of Sawford and Yeung ${ }^{12,13}$ of instationary homogeneous anisotropic turbulence in uniform shear flow at Taylor Reynolds numbers of about $150(\operatorname{Re} \approx 1500)$. Also here, asymmetry in damping can be shown to correspond to values of $b_{3}^{*}$ of around unity. ${ }^{29}$ The symmetric model for damping proposed by Thomson which becomes equal to Eq. (76) in case of homogeneous turbulence was found to give the best agreement with dispersion statistics obtained from DNS. ${ }^{13}$ In other words, also for anisotropic turbulence in uniform shear flow asymmetry in cross-correlations was found to be limited; its effect on dispersion small.

The aforementioned results of DNS and measurements all deal with cases of anisotropic shear induced turbulence. As such, they are relevant to shear induced turbulence in general. Limiting factors are the values of the Reynolds number Re. These were large to the extent that the Kolmogorov constant $C_{0}$ was around 4 . Yet larger values of Re are needed when aiming at data for values of $C_{0}$ which are closer to its asymptotic value of about 6 . In view of the complexities involved with the measurements and DNS at Re $\gg 1$, 
such data are not to be expected at short notice. Another approach would be to try to determine theoretically the nonunique terms from the equations, which govern fluid particle dynamics at microscopic level, i.e., the Navier-Stokes equations in Lagrangian representation. In this way one could try to derive expressions for relevant statistical quantities such as the cross-correlations of particle velocities for times shortly after marking. However, mathematical procedures will not be easy. The approach could pose questions, which are as difficult as those encountered in bridging the dynamics at microlevel and the models of macrolevel in statistical mechanics. ${ }^{36}$ The presented approach which rests on perturbation analysis using $C_{0}^{-1}$ as small parameter, supplemented with results of DNS and measurements to indicate truncation errors, provides an effective route toward a stochastic description which is sufficiently accurate for practical purposes.

\section{B. Diffusion limit}

The diffusion equation pertains to describing dispersion for times that are large after release of passive particles, i.e., $t \gg L u_{*}^{-1}$. At shorter times, predictions based on the diffusion equation may exhibit quite some error. It is inherent to the diffusion approximation. This has often been the motivation for using the Langevin equation rather than the diffusion equation to calculate dispersion. However, in the present case of turbulent flow, the diffusion equation may lead to errors even at large times after marking. As mentioned before, for general inhomogeneous forms of turbulence, there exists no physical limit process by which the diffusion approximation becomes exact. The diffusion approximation involves truncation of higher order derivatives whose contributions are supposed to be small in comparison with the second order derivative which models diffusion. ${ }^{3}$ In turbulence these higher order derivatives can only be disregarded because they can be shown to lead to contributions of $\mathcal{O}\left(C_{0}^{-2}\right)$ compared to the leading terms in the diffusion equation. Note that this truncation error occurs irrespective of and in addition to the contributions of relative magnitude $\mathcal{O}\left(C_{0}^{-2}\right)$ related to nonuniqueness and slight non-Gaussianity. The exact value of the truncation error can be calculated by comparing the long-time prediction of statistical parameters using the Langevin equation with that using the diffusion equation. A conservative testing case is the log-layer of wallinduced turbulence. ${ }^{29}$ Here, the mean energy dissipation rate $\varepsilon$ is a strongly varying function of distance $x_{2}$ to the wall [cf. Eq. (80)], causing slow convergence in the diffusion approximation. In Fig. 3, we have illustrated this by presenting the relative error in long-time prediction of the mean and standard deviation of particle displacement in wall-normal direction according to the diffusion equation in comparison with predictions using the Langevin equation. It is seen that there is still quite some error present when $C_{0}=6$. At the same time, it must be remembered that of all cases, the log-layer is presumably the one where convergence is slowest because of the strong change of $\varepsilon$ with distance from the wall. In general, the diffusion equation is a reasonably accurate tool for engineering applications.

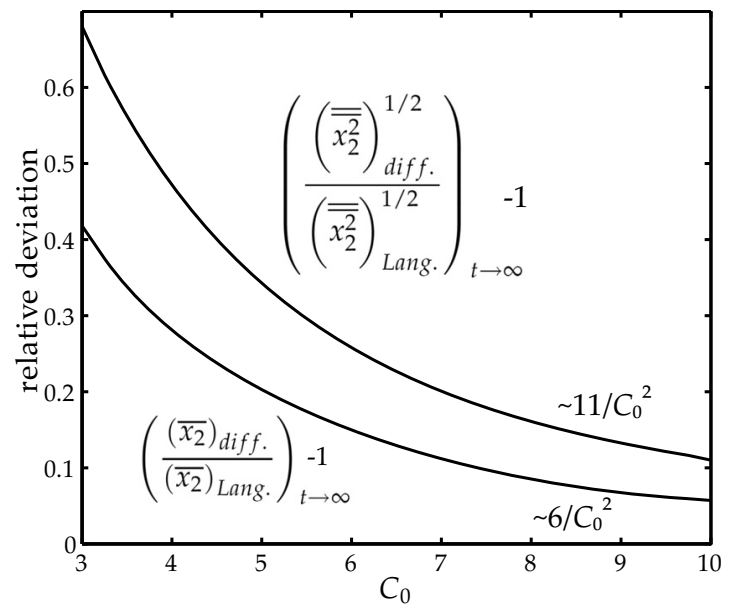

FIG. 3. Ratio of mean values and ratio of standard deviations of transverse fluid particle displacement $x_{2}$ minus 1 calculated from the diffusion equation and the long-time result of simulations of the Langevin equation for the log-layer of wall-induced turbulence vs Kolmogorov constant $C_{0}$. Overbar and double overbar denote Lagrangian-based mean and cumulant, respectively.

\section{Decaying turbulence}

The second term on the right hand side of Eqs. (75) and (76) describes the effect of changing Eulerian velocity statistics in the direction of the mean flow. Decaying turbulence behind a grid is a case where such behavior occurs in a pronounced manner. As mentioned before, for decaying isotropic Gaussian turbulence, exact solutions appropriate for the diffusion limit exist (see Appendix). Comparison of these solutions with those according to Eq. (75) reveals differences in the diffusion constants of only $1.2 \%$ for $C_{0}=6$ (see the last paragraph of Sec. VII).

\section{Non-Gaussian Eulerian velocity statistics}

One of the outcomes of the present analysis is that a slight Eulerian non-Gaussianity such that deviations from the Gaussian form of Eulerian velocities involve terms of $\mathcal{O}\left(C_{0}^{-1}\right)$, leads to alterations in dispersion statistics of $\mathcal{O}\left(C_{0}^{-2}\right)$. To indicate the effect of non-Gaussianity in further detail, consider the case of a one-dimensional slightly skewed distribution. The function $f_{c}$ in Eq. (61), which describes the deviation from Gaussianity, can then be represented by

$$
f_{c}=-\frac{1}{2} \kappa_{3} C_{0}\left(\frac{u^{\prime}}{\sigma^{1 / 2}}-\frac{1}{3} \frac{u^{\prime 3}}{\sigma^{3 / 2}}\right),
$$

where $\kappa_{3}$ is the skewness $\left\langle\left\langle u^{\prime 3}\right\rangle\right\rangle \sigma^{-3 / 2}, u^{\prime}$ is the onedimensional fixed-point Eulerian velocity, and $\sigma$ is the variance $\left\langle\left\langle u^{\prime 2}\right\rangle\right\rangle$. The contribution of non-Gaussianity in the damping term according to Eq. (63) is given by

$$
\frac{1}{2} \varepsilon \frac{d f_{c}}{d u^{\prime}}=\frac{1}{4} \frac{\kappa_{3} \varepsilon C_{0}}{\sigma^{3 / 2}}\left(u^{\prime 2}-\sigma\right) .
$$

This term is of the same form as the zero-mean quadratic term associated with covariance inhomogeneity, viz., $\frac{1}{2} \lambda_{j n}\left(\partial / \partial x_{m}\right) \sigma_{i j}\left(v_{m}^{\prime} v_{n}^{\prime}-\sigma_{m n}\right)$, a term which has been dropped in final result (76) because it contributes only to $\mathcal{O}\left(C_{0}^{-2}\right)$ in 
dispersion. The effect of covariance inhomogeneity has been assessed in some detail for the case of turbulent pipe flow. ${ }^{29}$ It was found to be small: corrections on dispersion statistics were minor and amounted to a few percent at most. The similarity between Eq. (83) and the term due to covariance inhomogeneity can be used to estimate the effect of skewed non-Gaussianity. Noting that $\varepsilon \sigma^{-3 / 2} \sim L^{-1}$, the two terms become of similar magnitude when $\frac{1}{4} \kappa_{3} C_{0} \approx \frac{1}{2}$, i.e., $\kappa_{3}=2 / C_{0}$. For $C_{0}=6$, this corresponds to a skewness of $1 / 3$, which is the value for which the effect of skewness is similar to that of the zero-mean quadratic term due to inhomogeneity. It indicates that for skewnesses of about 0.3 or less, its effect on dispersion statistics will be very minor. A similar conclusion is likely to be valid for kurtosis of about 0.3 or less. As mentioned in the final paragraph of Sec. VIII, the underlying reason that slight deviations from Gaussianity hardly effect dispersion is that the first three moments of the velocity distribution are fully captured by the Gaussian distribution [cf. Eq. (62)].

An interesting observation can be made concerning Gaussianity in the leading order formulation with respect to $C_{0}^{-1}$. As indicated in Sec. V, when $C_{0} \gg 1$, during a short time interval after particle marking, the dynamics of fluid particle velocity resemble those of particles obeying classical nondissipative Hamiltonian systems. From the results of statistical mechanics, it is known that the velocity distributions of such systems are Gaussian. It suggests that in the leading order formulation with respect to $C_{0}^{-1}$ also the velocity distributions of fluid particles must be Gaussian. Furthermore, as implied by results (25) and (27) to leading order in $C_{0}^{-1}$ and for the short time intervals considered, the Lagrangian velocity distributions are equal to the Eulerian distributions at the point of marking. The consequence is that also Eulerian velocity distributions must be Gaussian in the leading order formulation with respect to $C_{0}^{-1}$. According to this argumentation, Gaussianity of Eulerian velocities to leading order in $C_{0}^{-1}$ is not just a proposition motivated by experimental observation. It is a necessity in the present approach and a logical consequence of the connection with Hamiltonian dynamics when $C_{0}^{-1} \ll 1$.

\section{CONCLUDING REMARKS}

A derivation of the Langevin equation and diffusion equation of fluid particle in turbulent flow has been presented. The derivation is based on perturbation expansions in which the inverse of the Kolmogorov constant $C_{0}$ serves as small parameter. The expansions include the leading terms and next to leading terms with respect to $C_{0}^{-1}$; they involve a truncation error of $\mathcal{O}\left(C_{0}^{-2}\right)$ in dispersion statistics. In the derivation, it has been assumed that the probability distributions of fixed-point Eulerian fluid velocities are not far from Gaussian to the extent that skewness and kurtosis are about 0.3 or less.

Results presented enable the determination of the statistical parameters of fluid particle and passive admixture displacements in turbulent flow for the given values of fixedpoint statistics of the flow field, i.e., values of mean $u_{i}^{0}(\boldsymbol{x})$ and covariances $\sigma_{i j}(\boldsymbol{x})$ of fluid velocity and of mean energy dissipation rate $\varepsilon(\boldsymbol{x})$. The values of these Eulerian-based statistical parameters can be obtained from various sources covering a wide range of practical cases of turbulent flow. They can be calculated using commercially available numerical codes of computational fluid dynamics. Application of the presented Langevin equation [cf. Eq. (76)], supplemented with Eq. (77) to calculate the statistics of displacement, is expected to involve an error of a few percent. Dispersion calculated from the presented diffusion equation [cf. Eqs. (74) and (75)] involves a somewhat larger error. The presented equations provide the means to calculate and analyze turbulent dispersion of passive or almost passive admixture such as fumes, smoke, and aerosols in areas ranging from atmospheric fluid motion to flows in engineering devices.

\section{APPENDIX: EXACT SOLUTION FOR DECAYING ISOTROPIC TURBULENCE}

Isotropic turbulence is considered where particle dynamics in the three directions are statistically independent of each other. Attention is confined to the statistics of particle velocity $v^{\prime}$ and particle position $x^{\prime}$ in one direction only. Gaussianity of the Eulerian velocity field requires the damping term to be linear in velocity. A nonuniqueness problem does not exist in case of isotropy. ${ }^{11}$ The Fokker-Planck equation (23) becomes

$$
\begin{aligned}
\frac{\partial p}{\partial t^{\prime}}= & \frac{1}{2}\left(\frac{\varepsilon C_{0}}{\sigma}-\frac{1}{\sigma} \frac{d \sigma}{d t^{\prime}}\right) \frac{\partial}{\partial v^{\prime}}\left(v^{\prime} p\right)+\frac{1}{2} \varepsilon C_{0} \frac{\partial^{2} p}{\partial v^{\prime 2}} \\
& -\frac{\partial}{\partial x^{\prime}}\left(v^{\prime} p\right),
\end{aligned}
$$

where $p=p\left(v^{\prime}, x^{\prime}, t^{\prime}\right)$ is the joint probability density of $v^{\prime}$ and $x^{\prime}$ which varies with time $t^{\prime}$. As before, $v^{\prime}$ and $x^{\prime}$ are particle velocity and position in a frame that moves with the mean flow; in the present case, decaying turbulence behind a grid with mean velocity $u_{0}$. In Eq. (A1), $\sigma=\left\langle\left\langle u^{\prime 2}\right\rangle\right\rangle$, where $u^{\prime}$ is fluctuating fixed-point Eulerian velocity. According to the von Kármán-Howarth equation $\left(d / d t^{\prime}\right) \sigma=-\frac{2}{3} \varepsilon$ so that Eq. (A1) becomes

$$
\frac{\partial p}{\partial t^{\prime}}=\frac{1}{2} \frac{\varepsilon}{\sigma}\left(C_{0}+\frac{2}{3}\right) \frac{\partial}{\partial v^{\prime}}\left(v^{\prime} p\right)+\frac{1}{2} \varepsilon C_{0} \frac{\partial^{2} p}{\partial v^{\prime 2}}-\frac{\partial}{\partial x^{\prime}}\left(v^{\prime} p\right) .
$$

Here the fixed-point Eulerian averages $\varepsilon$ and $\sigma$ vary with time. According to the results of the asymptotic analysis of George, ${ }^{37}$ we have for large Re,

$$
\varepsilon=\varepsilon_{0}\left(t^{\prime} / t_{0}^{\prime}\right)^{-2}, \quad \sigma=\sigma_{0}\left(t^{\prime} / t_{0}^{\prime}\right)^{-1}, \quad \varepsilon_{0}=\frac{3}{2} \sigma_{0} t_{0}^{-1} .
$$

The solution to Eq. (A2) is the joint Gaussian distribution for $v^{\prime}$ and $x^{\prime 4}$. The distribution is fully specified once the mean squares of $v^{\prime}$ and $x^{\prime}$ and their correlation have been determined. To do so, we consider the fluctuation equations associated with Eq. (A2) (Ref. 28)

$$
\begin{aligned}
\frac{d v^{\prime}}{d t^{\prime}}= & -\frac{1}{2} \frac{\varepsilon_{0}}{\sigma_{0}}\left(C_{0}+\frac{2}{3}\right)\left(t^{\prime} / t_{0}^{\prime}\right)^{-1} v^{\prime} \\
& +\left[C_{0} \varepsilon_{0}\left(t^{\prime} / t_{0}^{\prime}\right)^{-2}\right]^{1 / 2} w\left(t^{\prime}\right),
\end{aligned}
$$




$$
\frac{d x^{\prime}}{d t^{\prime}}=v^{\prime}
$$

where $w\left(t^{\prime}\right)$ is Gaussian white noise of unit intensity. Now multiply Eq. (A4) with $v^{\prime}$ and average

$$
\frac{d \overline{v^{\prime 2}}}{d t}=-\frac{\varepsilon_{0}}{\sigma_{0}}\left(\frac{t^{\prime}}{t_{0}^{\prime}}\right)^{-1}\left(C_{0}+\frac{2}{3}\right) \overline{v^{\prime 2}}+C_{0} \varepsilon_{0}\left(\frac{t^{\prime}}{t_{0}^{\prime}}\right)^{-2},
$$

where as mentioned before the overbar denotes Lagrangian averaging. The solution of Eq. (A6) which satisfies the condition that $\overline{v^{\prime 2}}=\left\langle u_{0}^{\prime 2}\right\rangle=\sigma_{0}$ at $t^{\prime}=t_{0}^{\prime}$ is

$$
\overline{v^{\prime 2}}=\sigma_{0}\left(t^{\prime} / t_{0}^{\prime}\right)^{-1}=\left\langle\left\langle u^{\prime 2}\right\rangle\right\rangle \text {. }
$$

The evolution of the variance of particle velocity with time is the same as that of fixed-point Eulerian velocity.

Multiply Eq. (A4) with $x^{\prime}$ and average. Noting that

$$
\frac{d}{d t^{\prime}}\left(\overline{x^{\prime} v^{\prime}}\right)=\overline{v^{\prime} \frac{d x^{\prime}}{d t^{\prime}}}+\overline{x^{\prime} \frac{d v^{\prime}}{d t^{\prime}}}=\overline{v^{\prime 2}}+\overline{x^{\prime} \frac{d v^{\prime}}{d t^{\prime}}}
$$

one has

$$
\frac{d}{d t^{\prime}}\left(\overline{x^{\prime} v^{\prime}}\right)=-\frac{1}{2} \frac{\varepsilon_{0}}{\sigma_{0}}\left(\frac{t^{\prime}}{t_{0}^{\prime}}\right)^{-1}\left(C_{0}+\frac{2}{3}\right) \overline{x^{\prime} v^{\prime}}+\overline{v^{\prime 2}} .
$$

Implementing solution (A7) and requiring that $\overline{x^{\prime} v^{\prime}}=0$ at $t^{\prime}=t_{0}^{\prime}$, we obtain as solution for the correlation between $v^{\prime}$ and $x^{\prime}$,

$$
\overline{x^{\prime} v^{\prime}}=D_{1}\left\{1-\left(\frac{t^{\prime}}{t_{0}^{\prime}}\right)^{-(3 / 4) C_{0}-(1 / 2)}\right\}
$$

where $D_{1}$ is diffusivity defined as

$$
D_{1}=\frac{2 \sigma^{2}}{\varepsilon\left(C_{0}+\frac{2}{3}\right)} \text {. }
$$

To calculate the mean square of $x^{\prime}$, multiply Eq. (A5) with $x^{\prime}$ and average

$$
\frac{d \overline{x^{\prime 2}}}{d t^{\prime}}=2 \overline{x^{\prime} v^{\prime}}
$$

Upon substituting the solution for $\overline{x^{\prime} v^{\prime}}$ given by Eq. (A10), integrating, and requiring that $\overline{x^{\prime 2}}=0$ at $t^{\prime}=t_{0}^{\prime}$ (all particles start from the same position), one has

$$
\begin{aligned}
\overline{x^{\prime 2}}= & 2 D_{1} t^{\prime}\left[1+\left(\frac{3}{4} C_{0}-\frac{1}{2}\right)^{-1}\left(\frac{t^{\prime}}{t_{0}^{\prime}}\right)^{-(3 / 4) C_{0}-(1 / 2)}\right] \\
& -2 D_{1} t_{0}^{\prime}\left(\frac{3}{4} C_{0}+\frac{1}{2}\right)\left(\frac{3}{4} C_{0}-\frac{1}{2}\right)^{-1} .
\end{aligned}
$$

The joint Gaussian distribution of $v^{\prime}$ and $x^{\prime}$ is now specified by results (A7), (A10), and (A13). Note that for large times the distribution of $x^{\prime}$,

$$
G=\int_{-\infty}^{+\infty} p d v^{\prime}
$$

becomes equal to that described by the diffusion equation

$$
\frac{\partial G}{\partial t^{\prime}}=D_{1} \frac{\partial^{2} G}{\partial x^{\prime 2}},
$$

where $D_{1}$ is given by Eq. (A11).

${ }^{1}$ A. S. Monin and A. M. Yaglom, Statistical Fluid Mechanics (Dover, New York, 2007), Vol. 1.

${ }^{2}$ D. J. Thomson, "Criteria for the selection of stochastic models of particle trajectories in turbulent flows," J. Fluid Mech. 180, 529 (1987).

${ }^{3}$ J. J. H. Brouwers, "On diffusion theory in turbulence," J. Eng. Math. 44, 277 (2002).

${ }^{4}$ N. G. van Kampen, Stochastic Processes in Physics and Chemistry, 3rd ed. (Elsevier, New York, 2007).

${ }^{5}$ L. D. Landau and E. M. Lifshitz, Statistical Physics (Elsevier, New York, 2007).

${ }^{6} \mathrm{~S}$. R. de Groot and P. Mazur, Non-Equilibrium Thermodynamics (Dover, New York, 1984).

${ }^{7}$ L. E. Reichl, A Modern Course in Statistical Physics (Wiley-VCH, Weinheim, 2004).

${ }^{8}$ G. I. Taylor, "Diffusion by continuous movements," Proc. London Math. Soc. s2-20, 196 (1922)

${ }^{9}$ J. D. Wilson and B. L. Sawford, "Review of Lagrangian stochastic models for trajectories in the turbulent atmosphere," Boundary-Layer Meteorol. 78, 191 (1996).

${ }^{10}$ S. B. Pope, Turbulent Flows (Cambridge University Press, Cambridge, UK, 2000).

${ }^{11}$ M. S. Borgas and B. L. Sawford, "A family of stochastic models for two-particle dispersion in isotropic homogeneous stationary turbulence," J. Fluid Mech. 279, 69 (1994).

${ }^{12}$ B. L. Sawford and P. K. Yeung, "Eulerian acceleration statistics as a discriminator between Lagrangian stochastic models in uniform shear flow," Phys. Fluids 12, 2033 (2000).

${ }^{13}$ B. L. Sawford and P. K. Yeung, "Lagrangian statistics in uniform shear flow: Direct numerical simulation and Lagrangian stochastic models," Phys. Fluids 13, 2627 (2001).

${ }^{14}$ A. S. Monin and A. M. Yaglom, Statistical Fluid Mechanics (Dover, New York, 2007), Vol. 2.

${ }^{15}$ U. Frisch, Turbulence (Cambridge University Press, Cambridge, UK, 1998).

${ }^{16}$ G. A. Voth, A. La Porta, A. M. Crawford, J. Alexander, and E. Bodenschatz, "Measurements of particle accelerations in fully developed turbulence,” J. Fluid Mech. 469, 121 (2002).

${ }^{17}$ A. N. Kolmogorov, "Local structure of turbulence in an incompressible fluid at very high Reynolds number," Dokl. Akad. Nauk SSSR 30, 229 (1941).

${ }^{18}$ A. N. Kolmogorov, "A refinement of previous hypotheses concerning the local structure of turbulence in an incompressible fluid at very high Reynolds number," J. Fluid Mech. 13, 82 (1962).

${ }^{19}$ M. S. Borgas, "The multifractal Lagrangian nature of turbulence," Philos. Trans. R. Soc. London, Ser. A 342, 379 (1993).

${ }^{20}$ M. S. Borgas and B. L. Sawford, "Stochastic equations with multifractal random increments for modeling turbulent dispersion," Phys. Fluids 6, 618 (1994)

${ }^{21}$ A. A. Townsend, "The measurement of double and triple correlation derivatives in isotropic turbulence," Math. Proc. Cambridge Philos. Soc. 43, 560 (1947)

${ }^{22}$ J. Laufer, "The structure of turbulence in fully developed pipe flow," National Advisory Committee for Aeronaut Technical Report No. 1174, 1954 .

${ }^{23}$ J. F. Morrison, B. J. McKeon, W. Jiang, and A. J. Smits, "Scaling of the streamwise velocity component in turbulent pipe flow," J. Fluid Mech. 508, 99 (2004).

${ }^{24} \mathrm{P}$. S. Klebanoff, "Characteristics of turbulence in a boundary layer with zero pressure gradient," National Advisory Committee for Aeronaut Technical Report No. 1247, 1955.

${ }^{25}$ J. J. H. Brouwers, "Eulerian short-time statistics of turbulent flow at large Reynolds number," Phys. Fluids 16, 2300 (2004).

${ }^{26}$ J. J. H. Brouwers, "Dissipation equals production in the log layer of wallinduced turbulence," Phys. Fluids 19, 101702 (2007). 
${ }^{27}$ O. Bokhove and M. Oliver, "Parcel Eulerian-Lagrangian fluid dynamics of rotating geophysical flows," Proc. R. Soc. London, Ser. A 462, 2575 (2006).

${ }^{28}$ R. L. Stratonovich, Topics in the Theory of Random Noise (Gordon and Breach, New York, 1967), Vol. 1.

${ }^{29}$ J. J. H. Brouwers, "Langevin equation of a fluid particle in wall-induced turbulence," Theor. Math. Phys. 163, 677 (2010) [Teor. Mat. Fiz. 163, 328 (2010)]; Yu. S. Osipov, V. A. Sadovnichy, V. A. Matveev, and A. N. Sissakian, International Bogoliubov Conference of Russian Academy of Sciences, Dubna, Moscow, 21-27 August 2009.

${ }^{30}$ R. J. E. Walpot, C. W. M. van der Geld, and J. G. M. Kuerten, "Determination of the coefficients of langevin models for inhomogeneous turbulent flows by three-dimensional particle tracking velocimetry and direct numerical simulation," Phys. Fluids 19, 045102 (2007).

${ }^{31}$ J. G. M. Kuerten, "Subgrid modeling in particle-laden channel flow," Phys. Fluids 18, 025108 (2006)
${ }^{32}$ B. J. McKeon, J. Li, W. Jiang, J. F. Morrison, and A. J. Smits, "Further observations on the mean velocity distribution in fully developed pipe flow," J. Fluid Mech. 501, 135 (2004).

${ }^{33}$ R. Zhao and A. J. Smits, "Scaling of the wall-normal turbulence component in high-Reynolds-number pipe flow," J. Fluid Mech. 576, 457 (2007).

${ }^{34}$ P. A. Monkewitz, K. A. Chauhan, and H. M. Nagib, "Self-consistent highReynolds-number asymptotics for zero-pressure-gradient turbulent boundary layers," Phys. Fluids 19, 115101 (2007).

${ }^{35}$ S. Hoyas and J. Jiménez, "Scaling of the velocity fluctuations in turbulent channels up to $\mathrm{Re}_{\tau}=2003$," Phys. Fluids 18, 011702 (2006).

${ }^{36}$ J. L. Lebowitz, "From time-symmetric microscopic dynamics to timeasymmetric macroscopic behavior: An overview," e-print arXiv:0709.0724v1.

${ }^{37}$ W. K. George, "The decay of homogeneous isotropic turbulence," Phys. Fluids A 4, 1492 (1992). 